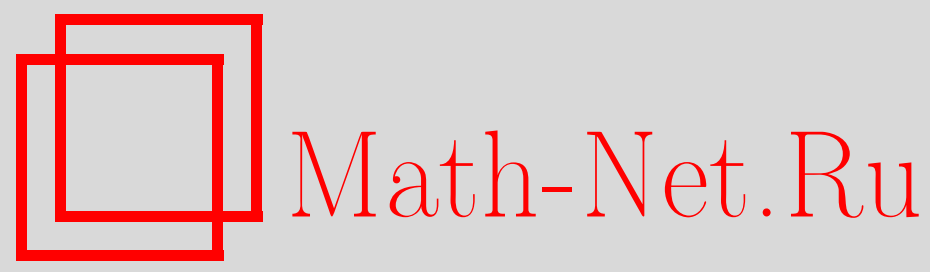

В. Е. Тараканов, А. М. Зубков, Цикловая структура степенных отображений в кольце классов вычетов, Дискрет. матем., 2013, том 25, выпуск 2, 39-62

DOI: https://doi.org/10.4213/dm1233

Использование Общероссийского математического портала Math-Net.Ru подразумевает, что вы прочитали и согласны с пользовательским соглашением http://www . mathnet.ru/rus/agreement

Параметры загрузки:

IP: 52.6 .47 .48

26 апреля 2023 г., 16:33:20

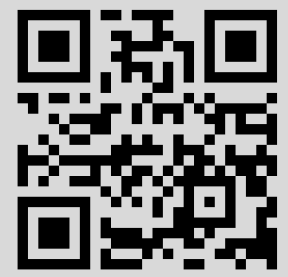




\title{
Цикловая структура степенных отображений в кольце классов вычетов
}

\author{
() 2013 г. В. Е. Тараканов, А. М. Зубков
}

\section{Введение}

В последние десятилетия в связи с применениями датчиков псевдослучайных чисел в криптографии повысился интерес к разработке и исследованию датчиков псевдослучайных чисел, которые, кроме простоты реализации и хороших статистических качеств выходной последовательности, обладают важными для криптографии свойствами, в частности, большим периодом, высокой сложностью восстановления начального значения по отрезку выходной последовательности, невозможностью прогнозирования будущих значений по известным и т. п.

В ряде работ (см., например, [2], [3], [4], [5], [8], [10], [11], [12]) изучаются датчики, основанные на операциях возведения в квадрат или в фиксированную степень по простому или специально подобранному составному модулю.

Taк, в [3] изучаются QR-генераторы, т. е. датчики, использующие операцию возведения в квадрат по модулю составного числа $N=p q$, где числа $p \neq q-$ простые и сравнимы с 3 по модулю 4 . Пусть $q_{N}(x)$ обозначает наименьший неотрицательный вычет числа $x^{2}$ по модулю $N$. Последовательность внутренних состояний QRгенератора с модулем $N$ строится по формуле

$$
x_{t+1}=\left\{\begin{array}{ll}
q_{N}\left(x_{t}\right), & \text { если } q_{N}(x)<N / 2, \\
N-q_{N}(x) & \text { в противном случае, }
\end{array} .\right.
$$

и в качестве выходной последовательности рассматривается

$$
y_{t}=q_{2}\left(x_{t}\right), \quad t=1,2, \ldots
$$

В [2] в качестве обоснования криптографических качеств порождаемой этим генератором последовательности используется сложность задачи извлечения квадратного корня в мультипликативной группе вычетов и задачи факторизации натуральных чисел. В [3] отмечается, что если $N=p q$, где числа $p \neq q-$ простые и сравнимы с 3 по модулю 4, то число квадратичных вычетов равно $\frac{1}{4}(p-1)(q-1)$ и в каждой паре $(x, N-x)$ оба числа не могут быть квадратичными вычетами. Поэтому формула (1) по сути означает, что множество квадратичных вычетов отображается в множество положительных вычетов, меньших $N / 2$. Значит, с точностью до этой 
замены свойства рекуррентной последовательности (1) совпадают со свойствами последовательности

$$
x_{t+1}=x_{t}^{2}(\bmod N), \quad t=0,1, \ldots
$$

Цикловая структура отображений вида $x \rightarrow x^{d}(\bmod N)$ для случаев, когда $d \geqslant 2$ и $N$ - простое число, изучалась в [5], [11], а в [10] рассмотрен случай, когда $N=p^{m}$ - степень простого числа $p$. В частности, в [10] показано, что для числа $C_{r}\left(d, p^{m}\right)$ циклов длины $r$ отображения $x \rightarrow x^{d}\left(\bmod p^{m}\right)$ справедливы формулы

$$
\begin{gathered}
C_{r}\left(d, p^{m}\right)=\frac{1}{r} \sum_{j: j \mid r} \mu(j) \text { НОД }\left(p^{m}-p^{m-1}, d^{r / j}-1\right), \\
\sum_{r \geqslant 1} C_{r}\left(d, p^{m}\right)=\sum_{j: j \mid \rho} \frac{\varphi(j)}{\operatorname{ord}_{j} d},
\end{gathered}
$$

где $\mu(j)$ и $\varphi(j)$ - функции Мебиуса и Эйлера соответственно, $\operatorname{ord}_{j} d$ при НОД $(d, j)=$ 1 - порядок числа $d$ по модулю $j$, а число $\rho$ определяется условиями $d=$ $p_{1}^{n_{1}} p_{2}^{n_{2}} \ldots p_{s}^{n_{s}}\left(p_{1}, \ldots, p_{s}\right.$ - простые $), p^{m}-p^{m-1}=p_{1}^{r_{1}} \ldots p_{s}^{r_{s}} \rho$, НОД $(d, \rho)=1$. В [4] приведено количественное описание цикловой структуры отображения $x \rightarrow x^{d}(\bmod N)$ на множестве обратимых элементов кольца $\mathbb{Z}_{N}$ при любых $N$ и $d$, использующее, в том числе, мультипликативную структуру числа циклических точек отображения, и для случая, когда $N$ есть произведение двух простых чисел, изучается доля числа циклических точек, лежащих на коротких циклах или на циклах, длины которых не делятся на число, однозначно определяемое по $N$.

Результатом настоящей работы является конструктивное описание структуры множества циклов отображений $x \rightarrow x^{d}(\bmod N)$ для произвольных значений $d$ и $N$. В $\S 1-2$ излагается комбинаторно-алгебраический подход к описанию этих цикловых структур. Он позволяет свести задачу к двум следующим: а) описанию цикловых структур степенных отображений на произвольных примарных циклических группах и б) нахождению цикловой структуры отображения на прямом произведении таких групп по известным цикловым структурам отображения на сомножителях. Описание цикловой структуры отображения $f^{(d)}: x \rightarrow x^{d}$ для примарных циклических групп нечетного порядка содержится в $\S 3$, а для групп четного порядка - в 4. Наконец, в $\S 5$ с помощью введенной в 22 операции композиции цикловых структур и результатов, полученных в $\S 3-4$, дается описание цикловых структур отображений $f^{(d)}$ на множествах $\mathbb{Z}_{N}^{*}$ обратимых элементов кольца $\mathbb{Z}_{N}$ классов вычетов по модулю $N$ для любых $d, N \geqslant 2$.

\section{1. Комбинаторно-алгебраический подход к изуче- нию цикловой структуры степенных отображе- ний на кольце $\mathbb{Z}_{N}$}

Для натурального $N$ обозначим через $\mathbb{Z}_{N}$ кольцо классов вычетов по модулю $N$. Элементы $\mathbb{Z}_{N}$ представляем числами $0,1, \ldots, N-1$. Через $\mathbb{Z}_{N}^{*}$ обозначим множество всех чисел из $\mathbb{Z}_{N}$, взаимно простых с $N$. 
Для любого натурального $d \geqslant 2$ равенство $f^{(d)}(x)=x^{d}(\bmod N)$ определяет отображение $f_{N}^{(d)}: \mathbb{Z}_{N} \rightarrow \mathbb{Z}_{N}$, которому соответствует ориентированный граф $G_{N}^{d}$ с множеством вершин $V_{N}=\{0,1, \ldots, N-1\}$ и множеством ребер $E_{N}^{(d)}=\left\{\left(x, f_{N}^{(d)}(x)\right)\right.$ : $\left.x \in \mathbb{Z}_{N}\right\}$.

$\Gamma$ раф $G_{N}^{d}$ разбивается на связные компоненты, каждая из которых состоит из цикла с подходами. Если граф $G_{N}^{d}$ состоит из $k_{1}$ компонент с циклами длины $l_{1}, \ldots$, $k_{r}$ компонент с циклами длины $l_{r}$, то будем говорить, что граф $G_{N}^{d}$ имеет цикловую структуру

$$
\operatorname{Str}\left(f_{N}^{(d)}, \mathbb{Z}_{N}\right)=\left(l_{1}\right)^{k_{1}} \ldots\left(l_{r}\right)^{k_{r}}=\prod_{i=1}^{r}\left(l_{i}\right)^{k_{i}} .
$$

Аналогичное обозначение будем использовать для цикловой структуры произвольного отображения конечного множества в себя.

\section{1. Вспомогательные результаты}

Напомним вначале ряд хорошо известных фактов из теории чисел и теории конечных абелевых групп.

Предложение $\mathbf{A}$ (китайская теорема об остатках, [6]). Пусть $N=p_{1}^{v_{1}} p_{2}^{v_{2}} \ldots p_{m}^{v_{m}}-$ каноническое разложение натурального числа $N$ в произведение степеней простых чисел. Существует взаимно однозначное отображение

$$
\mathbb{Z}_{N} \leftrightarrow \mathbb{Z}_{p_{1}^{v_{1}}} \times \mathbb{Z}_{p_{2}^{v_{2}}} \times \ldots \times \mathbb{Z}_{p_{m}^{v_{m}}}
$$

при котором любому элементу а $\in \mathbb{Z}_{N}$ соответствует вектор $\left(a_{1}, a_{2}, \ldots, a_{m}\right), a_{i} \in \mathbb{Z}_{p_{i}^{v_{i}}}, i=1,2, \ldots, m$, дде $a_{1}, \ldots, a_{m}$ - наименьшие неотрицательные вычеты а по модулям $p_{1}^{v_{1}}, \ldots, p_{m}^{v_{m}}$ соответственно, и обратно, каждому такому вектору соответствует элемент $a \in \mathbb{Z}_{N}$, для которого $a_{i} \equiv a$ $\left(\bmod p_{i}^{v_{i}}\right), i=1,2, \ldots, m$.

Мы фиксируем такое соответствие и, когда это удобно, записываем элемент $a \in$ $\mathbb{Z}_{n}$ в виде $\left(a_{1}, a_{2}, \ldots, a_{m}\right)$. Из соответствия (3) вытекает аналогичная связь между $\mathbb{Z}_{N}^{*}$ и $\mathbb{Z}_{p_{i}^{v_{i}}}^{*}, i=1, \ldots, m$.

Предложение В. Пусть $N=\prod_{i=1}^{m} p_{i}^{v_{i}}-$ каноническое разложение натуралъного числа $N$.

а) Совокупность $\mathbb{Z}_{N}^{*}$ всех обратимых элементов кольца $\mathbb{Z}_{N}$ образует группу по умножению.

б) Имеет место изоморфизм:

$$
\mathbb{Z}_{N}^{*} \cong \mathbb{Z}_{p_{1}^{v_{1}}}^{*} \times \mathbb{Z}_{p_{2}^{v_{2}}}^{*} \times \ldots \times \mathbb{Z}_{p_{m}^{v_{m}}}^{*}
$$

если при этом $a=\left(a_{1}, \ldots, a_{m}\right), b=\left(b_{1}, \ldots, b_{m}\right) \in \mathbb{Z}_{N}^{*}$, mо $a b=\left(a_{1} b_{1}, \ldots, a_{m} b_{m}\right)$.

в) Порядок $\left|\mathbb{Z}_{N}^{*}\right|$ группь $\mathbb{Z}_{N}^{*}$ равен $\varphi(N)=\prod_{i=1}^{m}\left(p_{i}^{v_{i}}-p_{i}^{v_{i}-1}\right)(\varphi-$ функиия Эйлера).

Циклическую группу по умножению с $n$ элементами обозначаем $C_{n}$.

Предложение С. Пусть $G$ - конечная абелева группа порядка $n$ u $n=\prod_{i=1}^{k} u_{i}^{\alpha_{i}}$, где $u_{1}, \ldots, u_{k}$ - попарно различные простые числа, $\alpha_{1}, \ldots, \alpha_{k}>0$. Тогда имеет место изоморфизм:

$$
G \cong C_{u_{q}^{\alpha_{q}}} \times C_{u_{2}^{\alpha_{2}}} \times \ldots \times C_{u_{k}^{\alpha_{k}}}
$$


Следующее предложение описывает подгруппы циклических групп.

Предложение D. Пусть $G=\langle g\rangle=C_{n}$ - конечная ииклическая группа порядка $n$, порождаемая элементом $g$.

а) Любая подгруппа $H \subseteq G$ группы $G$ есть ииклическая группа и её порядок есть делитель $n$.

б) Для любого делителя $r$ числа $n$ в $G$ существует единственная подгруппа $H$ порядка $r$; она порождается элементом $g^{n / r}: H=\left\langle g^{n / r}\right\rangle$.

Для отображения $\psi$ конечного множества $S$ в себя назовём подстановочным ядpoм $\operatorname{Ker}(\psi, S)$ множество всех элементов $S$, принадлежащих циклам графа отображения $\psi$. Отображение $\psi$ взаимно однозначно (т. е. является подстановкой) на $\operatorname{Ker}(\psi, S)$ и не взаимно однозначно на любом более широком подмножестве. Укажем некоторые свойства $\operatorname{Ker}(\psi, S)$ в случае, когда $\psi-$ степенное отображение на $S=G$, где $G$ - конечная абелева группа.

Предложение 1. Пусть $G$ - конечная абелева группа и $G(d)-$ совокупность всех её элементов, порядок которых взаимно прост с данным числом $d \geqslant 2$. Тогда:

а) $G(d)$ - әрynna;

б) отображение $f: x \rightarrow x^{d}-$ автоморфизм группь $G(d)$;

в) $\operatorname{Ker}(f, G)=G(d)$.

Аналогичное утверждение для случая, когда $G=\mathbb{Z}_{N}^{*}$, содержится в Теореме 1 из [4].

Доказательство. а) Пусть $a_{1}, a_{2} \in G(d)$. Тогда порядок $a_{1} a_{2}$ также взаимно прост с $d$, так как он является делителем наименьшего общего кратного порядков элементов $a_{1}$ и $a_{2}$. Если $a \in G(d)$, то $a^{-1}$ также принадлежит $G(d)$. Таким образом, $G(d)-$ группа.

б) Пусть $e$ - единица группы $G$. Тогда $f(e)=e$, и если $a \in G(d)$, то из $f(a)=$ $a^{d}=e$ следует, что $a=e$. Покажем, далее, что если $a \in G(d), a \neq e$, имеет порядок $m>1,(m, d)=1$, то существует единственный элемент $b \in G(d)$, для которого $f(b)=a$, и он имеет вид $b=a^{x}, 0<x<m$.

Действительно, мы можем определить $x$ из сравнения $x d \equiv 1(\bmod m)$, которое, ввиду условия $(m, d)=1$, имеет единственное решение $x \in\{1,2, \ldots, m-1\}$.

Предположим теперь, что $f(c)=a$ для некоторого $c \in G(d)$. Тогда $c^{d}\left(a^{-x}\right)^{d}=e$, т. е. $\left(c a^{-x}\right)^{d}=e$. Но в п. а) мы показали, что $G(d)-$ группа, следовательно, $c a^{-x}$ имеет порядок, взаимно простой с $d$. Поэтому должно быть $c a^{-x}=e$ и $c=a^{x}=b$. Таким образом, отображение $f$ взаимно однозначно на группе $G(d)$. Кроме того, $f\left(a_{1} a_{2}\right)=f\left(a_{1}\right) f\left(a_{2}\right)$ для $a_{1}, a_{2} \in G(d)$, и мы получаем, что $f$ - автоморфизм группы $G(d)$.

в) В п. б) мы фактически показали, что $G(d) \subseteq \operatorname{Ker}(f, G)$. Докажем теперь, что $G(d)=\operatorname{Ker}(f, G)$.

Допустим противное: $x \in \operatorname{Ker}(f, G)$, но $x \notin G(d)$. Тогда порядок $x$ делится на некоторый отличный от 1 делитель $d^{\prime}$ числа $d$. Следовательно, порождаемая $x$ циклическая группа $\langle x\rangle$ содержит, согласно Предложению D, циклическую подгруппу $\langle b\rangle, b \neq e$, порядка $d^{\prime}$, т. е. $b^{d^{\prime}}=e$ и, следовательно, $f(b)=b^{d}=e$. Так как $b$ есть степень $x$, то $b$ принадлежит некоторому циклу, т. е. $b \in \operatorname{Ker}(f, G)$. Kaк мы заметили выше, также $e \in \operatorname{Ker}(f, G)$. Таким образом $f(b)=f(e)=e$ для двух различных элементов $b, e \in \operatorname{Ker}(f, G)$. Но, по определению, $f$ должно быть взаимно 
однозначным на $\operatorname{Ker}(f, G)$. Это противоречие показывает, что $\operatorname{Ker}(f, G)=G(d)$. Предложение доказано.

Следствие 1. Пусть $G$ - прямое произведение примарных ииклических групп:

$$
G=\prod_{p \in \mathrm{P}} C_{p^{v_{p}}}, \quad v_{p}>0
$$

где $\mathrm{P}$ - конечный набор простых чисел. Если на $G$ действует степенное отображение $f^{(d)}, d \geqslant 2$, то

$$
\operatorname{Ker}\left(f^{(d)}, G\right)=\prod_{p \in \mathrm{P}: p \nmid d} C_{p^{v_{p}}}
$$

если при этом $\mathrm{P}$ не содержит простых чисел, не делящих $d, \operatorname{mo} \operatorname{Ker}\left(f^{(d)}, G\right)=C_{1}$ состоит из единственного элемента е (единищы группы $G$ ).

Замечание 1. Если $N-$ составное число и $N=p_{1}^{v_{1}} p_{2}^{v_{2}} \ldots p_{m}^{v_{m}}-$ его разложение на простые множители, то граф $G_{N}^{d}$ отображения $f^{(d)}(x)=x^{d}(\bmod N)$ распадается на $2^{m}$ изолированных подграфов $G_{N}^{d}\left(\varepsilon_{1}, \ldots, \varepsilon_{m}\right), \varepsilon_{1}, \ldots, \varepsilon_{m} \in\{0,1\}$, где множество $V_{N}\left(\varepsilon_{1}, \ldots, \varepsilon_{m}\right)$ вершин подграфа $G_{N}^{d}\left(\varepsilon_{1}, \ldots, \varepsilon_{m}\right)$ состоит из всех таких $x \in \mathbb{Z}_{N}$, что $x$ делится на $p_{j}$ тогда и только тогда, когда $\varepsilon_{j}=1, j \in\{1, \ldots, m\}$. Цикловая структура ограничения $f_{N}^{(d)}$ на $V_{N}\left(\varepsilon_{1}, \ldots, \varepsilon_{m}\right)$ совпадает с цикловой структурой отображения $f_{N\left(\varepsilon_{1}, \ldots, \varepsilon_{m}\right)}^{(d)}$, где $N\left(\varepsilon_{1}, \ldots, \varepsilon_{m}\right)=\prod_{j=1}^{m} p_{j}^{\varepsilon_{j} v_{j}}$. Поэтому в дальнейшем основное внимание будет уделяться цикловой структуре отображения $f_{N}^{(d)}$ на мультипликативной абелевой группе $\mathbb{Z}_{N}^{*}$, состоящей из множества $V_{N}(0, \ldots, 0)$ всех вычетов, взаимно простых с $N$, т. е. обратимых элементов кольца $\mathbb{Z}_{N}$.

\section{2. Автоморфизмы примарных циклических групп и степен- ные отображения}

Как мы установим далее, цикловая структура степенного отображения $f^{(d)}$ на $\mathbb{Z}_{N}^{*}$ полностью определяется цикловыми структурами вида $\operatorname{Str}\left(f^{(d)}, C_{p^{\alpha}}\right)$, где $C_{p^{\alpha}}-$ произвольная примарная циклическая группа ( $p$ - простое, $\alpha$ - целое число). Обозначим через $\operatorname{pm}\left(C_{n}\right)$ совокупность всех отображений $f^{(d)}$, где $d<n$ и взаимно просто c $n$.

В этом разделе мы остановимся на связи степенных отображений с автоморфизмами этих групп. Группу всех автоморфизмов группы $G$ обозначаем Aut $G$. Строение группы Aut $G$ для примарной циклической группы $G$ определяется следующим образом (см. [9]).

Предложение Е. Пусть $p-$ простое число, $\alpha \geqslant 1-$ целое.

а) Если р нечетно, то Aut $C_{p^{\alpha}}$ - ииклическая группа порядка $p^{\alpha}-p^{\alpha-1}$.

б) $Е с л и ~ p=2, \alpha \geqslant 2$, то Aut $C_{2^{\alpha}}$ изоморфна прямому произведению:

$$
\text { Aut } C_{2^{\alpha}} \cong C_{2} \times C_{2^{\alpha-2}}
$$

если $\alpha=1$, то Aut $C_{2}=\{\varepsilon\}(\varepsilon-$ тождественное отображение $)$. 
Рассматриваем действие $f^{(d)}$ на группы $C_{n}$. Ясно, что оно зависит только от наименьшего неотрицательного вычета числа $d$ по модулю $n$. Поэтому можно считать, что $d<n$. На $\mathrm{pm}\left(C_{n}\right)$ определим операцию о как последовательное выполнение отображений $f^{\left(d_{1}\right)}$ и $f^{\left(d_{2}\right)}$ из $\operatorname{pm}\left(C_{n}\right): f^{\left(d_{1}\right)} \circ f^{\left(d_{2}\right)}=f^{\left(d_{3}\right)}$, где $d_{3} \equiv d_{1} d_{2}(\bmod n)$, $d_{3}<n$. Для $f^{(d)} \in \operatorname{pm}\left(C_{n}\right)$ определяется обратное отображение: $\left(f^{(d)}\right)^{-1}=f^{\left(d^{-1}\right)}$, где $d^{-1}<n-$ решение сравнения $x d \equiv 1(\bmod n)$.

Ясно, что относительно операции о совокупность $\operatorname{pm}\left(C_{n}\right)$ - группа с единицей $f^{(1)}=\varepsilon$; назовем её группой степеннъх отображений.

Предложение 2. Группа степенных отображений $\operatorname{pm}\left(C_{n}\right)$ изоморфна группе $\mathbb{Z}_{n}^{*}$.

Утверждение очевидно.

Рассмотрим теперь группы степенных отображений для примарных циклических групп $C_{p^{\alpha}}$. Порождающий элемент группы $C_{p^{\alpha}}$ обозначаем через $g$, единицу - через $e$.

Предложение 3. Для всякого простого р и иелого $\alpha \geqslant 1$ группа $\mathrm{pm}\left(C_{p^{\alpha}}\right)$ изоморфна әpynne Aut $C_{p^{\alpha}}$.

Доказательство. Всякое отображение $f^{(d)} \in \mathrm{pm}\left(C_{p^{\alpha}}\right)$ взаимно однозначно и удовлетворяет соотношениям

$$
f^{(d)}\left(x_{1} x_{2}\right)=f^{(d)}\left(x_{1}\right) f^{(d)}\left(x_{2}\right), \quad x_{1}, x_{2} \in C_{p^{\alpha}}, \quad f^{(d)}(e)=e,
$$

т. е. оно порождает автоморфизм группы $C_{p^{\alpha}}$. Обозначим его $\psi_{d}$. Ясно также, что произведению $f^{\left(d_{1}\right)} \circ f^{\left(d_{2}\right)}$ отвечает автоморфизм $\psi_{d_{1}} \psi_{d_{2}}=\psi_{d_{3}}$, где $d_{3} \equiv d_{1} d_{2}(\bmod$ $\left.p^{\alpha}\right), d_{3}<p^{\alpha}$. Автоморфизм $\psi_{d}$ при $d \neq 1$ не может быть тождественным: из $\psi_{d}=\varepsilon$ следовало бы $\psi_{d}(g)=g^{d}=g$ и $g^{d-1}=e$, что невозможно, так как $0<d-1<p^{\alpha}$, а $g^{k} \neq e$, если $0<k<p^{\alpha}$. Таким образом, мы получаем изоморфное вложение: $\operatorname{pm}\left(C_{p^{\alpha}}\right) \subseteq \operatorname{Aut} C_{p^{\alpha}}$.

С другой стороны, если $\psi$ - любой автоморфизм группы $C_{p^{\alpha}}=\langle g\rangle$, то $\psi(g)=g^{d}$, и $g^{d}$ должен также быть порождающим элементом. Поэтому $d$ не делится на $p$. Далее, для любого $h=g^{a} \in C_{p^{\alpha}}$ имеем $\psi(h)=\psi\left(g^{a}\right)=(\psi(g))^{a}=\left(g^{d}\right)^{a}=h^{d}$. Следовательно, $\psi=\psi_{d}, p \nmid d$; при этом $\psi=\varepsilon$ равносильно $d=1$. Тем самым получаем противоположное изоморфное вложение: Aut $C_{p^{\alpha}} \subseteq \operatorname{pm}\left(C_{p^{\alpha}}\right)$. В итоге приходим к изоморфизму: $\operatorname{pm}\left(C_{p^{\alpha}}\right) \cong \operatorname{Aut} C_{p^{\alpha}}$. Предложение доказано.

Следствие 2. Всякое степенное отображение $f^{(d)}$ на группе $C_{p^{\alpha}}$, где $p-$ нечётное простое, $\alpha \geqslant 1, p \nmid d$, есть степень некоторого отображения $f^{\left(d_{1}\right)}$, где $d_{1}-$ элемент порядка $p^{\alpha}-p^{\alpha-1}$ в группе $\mathbb{Z}_{p^{\alpha}}^{*}$.

Следствие 3. Всякое степенное отображение $f^{(d)}$ на группе $C_{2^{\alpha}}$, где $\alpha \geqslant 3 u d$ нечётно, представляется в виде произведения $\left(f^{\left(d_{1}\right)}\right)^{w} \circ\left(f^{\left(d_{2}\right)}\right)^{s}, 0 \leqslant w<2^{\alpha-2}, s=$ 0 или $1, u d_{1}, d_{2} \in \mathbb{Z}_{2^{\alpha}}^{*}$ - элементы порядков $2^{\alpha-2}$ и 2 соответственно.

Нетрудно заметить, что при $\alpha \geqslant 3$ в группе $\mathbb{Z}_{2^{\alpha}}^{*} \cong\left\langle d_{1}\right\rangle \times\left\langle d_{2}\right\rangle$, где $d_{1}, d_{2}$ - элементы порядков, соответственно, $2^{\alpha-2}$ и 2 , в качестве $d_{2}$ всегда можно взять $2^{\alpha}-1$, т. е. каждый элемент $\mathbb{Z}_{2^{\alpha}}^{*}$ записывать в виде $\pm d_{1}^{w}, 0 \leqslant w<2^{\alpha-2}$. 
Мы можем теперь при любых $d$ и $N$ описать алгебраическое строение подстановочного ядра $\operatorname{Ker}\left(f^{(d)}, \mathbf{Z}_{N}^{*}\right)$ отображения $f^{(d)}$, т. е. максимального подмножества в $\mathbb{Z}_{N}^{*}$, на котором $f^{(d)}$ действует как подстановка. Пусть

$$
N=2^{\alpha_{0}} p_{1}^{\alpha_{1}} \ldots p_{m}^{\alpha_{m}} \text {, где } \alpha_{0}=0 \text {, если } N \text { нечётно, }
$$

- каноническое разложение для $N$, пусть $L_{i}=\left(p_{i}-1\right) p_{i}^{\alpha_{i}-1}$ и

$$
L_{i}=\prod_{j=1}^{r_{i}} u_{i j}^{\alpha_{i j}}, \quad u_{i j}-\text { простые числа, } \alpha_{i j}>0 \text {, }
$$

— каноническое разложение для $L_{i}>1$, a

$$
U_{i}(d)=\left\{u_{i 1}, u_{i 2}, \ldots, u_{i r_{i}}\right\}, \quad i=1, \ldots, m,
$$

- множество всех простых и не делящих $d$ делителей $L_{i}$, а $U_{0}(d)=\{2\}$ только при $\alpha_{0} \geqslant 2$ и нечетном $d$, в остальных случаях $U_{0}(d)=\varnothing$.

Предложение 4. Пусть $d \geqslant 2, f^{(d)}: x \rightarrow x^{d}$-отображение, определенное на $\mathbb{Z}_{N}^{*}$, $N$ - число из (4). Тогда $\operatorname{Ker}\left(f^{(d)}, \mathbb{Z}_{N}^{*}\right)$ представляется в виде прямого произведения примарных ииклических групп:

$$
\operatorname{Ker}\left(f^{(d)}, \mathbb{Z}_{N}^{*}\right) \cong \begin{cases}C\left(d, \alpha_{0}\right) \prod_{i=1}^{m}\left(\prod_{u_{i j} \in U_{i}(d)} C_{u_{i j}^{\alpha_{i j}}}\right), & \text { если } U(d) \neq \varnothing, \\ C_{1}, & \text { если } U(d)=\varnothing\end{cases}
$$

где $u_{i j}, \alpha_{i j}$ - числа из формуль $(5), C(d, n)=C_{2} \times C_{2^{n-2}}$ при $n \geqslant 2$ и нечетном $d$, в остальных случаях $C(d, n)=C_{1}, U(d) \stackrel{\text { def }}{=} \bigcup_{i=0}^{m} U_{i}(d)$, а $U_{i}(d)-$ множества из (6).

Доказательство. Из Предложения В следует, что $\mathbb{Z}_{N}^{*} \cong \prod_{i=0}^{m} \mathbb{Z}_{p_{i}^{\alpha_{i}}}^{*}$ (здесь $\left.p_{0}=2\right)$.

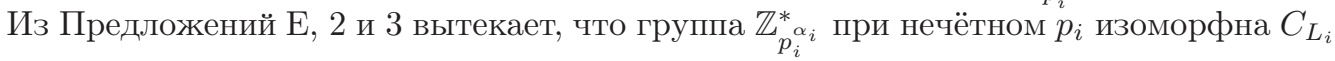
для $L_{i}$ из (5). Применяя затем Предложение $\mathrm{C}$, мы получаем, что $C_{L_{i}}-$ прямое произведение примарных циклических групп:

$$
\mathbb{Z}_{p_{i}^{\alpha_{i}}}^{*} \cong C_{L_{i}} \cong \prod_{j=1}^{r_{i}} C_{u_{i j}^{\alpha_{i j}}}, \quad i=1,2, \ldots, m
$$

где $u_{i j}, \alpha_{i j}, r_{i}$ определены в (5).

Аналогично,

$$
\mathbb{Z}_{2^{n}}^{*} \cong \begin{cases}C_{2} \times C_{2^{n-2}}, & n \geqslant 2, \\ C_{1}, & n=1 .\end{cases}
$$

Следовательно, при любых $d, N \geqslant 2$ группа $\mathbb{Z}_{p_{i}^{\alpha_{i}}}^{*}$ изоморфна прямому произведению циклических групп вида (8) или (9). Далее, используя Предложение 1, нетрудно заметить, что

$$
\operatorname{Ker}\left(f^{(d)}, Z_{N}^{*}\right)=\mathbb{Z}_{N}^{*}(d) \cong \prod_{i=0}^{m} \mathbb{Z}_{p_{i}^{\alpha_{i}}}^{*}(d)=\prod_{i=0}^{m} \operatorname{Ker}\left(f^{(d)}, \mathbb{Z}_{p_{i}^{\alpha_{i}}}^{*}\right)
$$


Если $U_{0}(d) \neq \varnothing$, то $\operatorname{Ker}\left(f^{(d)}, \mathbb{Z}_{2^{\alpha_{0}}}^{*}\right)=C\left(d, \alpha_{0}\right)$ в силу $(9)$. Наконец, по Следствию $1, \operatorname{Ker}\left(f^{(d)}, \mathbb{Z}_{p_{i}^{\alpha_{i}}}^{*}\right) \cong \prod_{u_{i j} \in U_{i}(d)} C_{u_{i j}^{\alpha_{i j}}}, i=1, \ldots, m$. Итак,

$$
\operatorname{Ker}\left(f^{(d)}, \mathbb{Z}_{N}^{*}\right) \cong C\left(d, \alpha_{0}\right) \prod_{i=1}^{m}\left(\prod_{u_{i j} \in U_{i}(d)} C_{u_{i j}^{\alpha_{i j}}}\right)
$$

если хотя бы одно из множеств $U_{i}(d), i=0,1, \ldots, m$, не пусто, и $\operatorname{Ker}\left(f^{(d)}, \mathbb{Z}_{N}^{*}\right)=C_{1}$, если $U(d)=\bigcup_{i=0}^{m} U_{i}(d)=\varnothing$.

Предложение доказано.

Таким образом, мы сможем найти цикловую структуру отображения $f^{(d)}$ на $\mathbb{Z}_{N}^{*}$, если:

a) найдем формулу для $\operatorname{Str}\left(f^{(d)}, C_{u^{\alpha}}\right)$, где $u$ - простое число, не делящее $d$, $\alpha \geqslant 1$, и

б) укажем правило нахождения $\operatorname{Str}\left(f^{(d)}, C_{u_{1}^{\alpha_{1}}} \times \ldots \times C_{u_{r}^{\alpha_{r}}}\right)$ по $\operatorname{Str}\left(f^{(d)}, C_{u_{i}^{\alpha_{i}}}\right)$, $i=1, \ldots, r$.

Рассмотрим сперва задачу б).

\section{2. Композиция цикловых структур}

Пусть $S$ - конечное множество, $\psi$ - подстановка на $S$, имеющая $k_{1}$ циклов длины $l_{1}$, $k_{2}$ циклов длины $l_{2}, \ldots, k_{r}$ циклов длины $r, k_{1} l_{1}+k_{2} l_{2}+\ldots+k_{r} l_{r}=|S|$. Цикловую структуру $\psi$ обозначаем, как и раньше, через

$$
\operatorname{Str}(\psi, S)=\left(l_{1}\right)^{k_{1}}\left(l_{2}\right)^{k_{2}} \ldots\left(l_{r}\right)^{k_{r}}=\prod_{i=1}^{r}\left(l_{i}\right)^{k_{i}}
$$

при этом $(l)^{k}$ называем фрагментом $\operatorname{Str}(\psi, S)$, если он совпадает с одним из $\left(l_{i}\right)^{k_{i}}$, $i=1,2, \ldots, r$.

Пусть $\mathrm{H}=\left(H_{1}, \ldots, H_{M} ; \pi_{1}, \ldots, \pi_{M}\right)$ - система из $M$ конечных множеств и действующих на них подстановок $\pi_{r}, r=1, \ldots, M$. Мы будем рассматривать системы, удовлетворяющие условию

$$
\pi_{r}(x)=\pi_{s}(x) \quad \text { для всех } x \in H_{r} \cap H_{s} \text { и } 1 \leqslant r<s \leqslant M .
$$

Если множества $H_{r}$ в системе Н попарно не пересекаются, то такая система удовлетворяет условию (10). Другим интересным для нас примером систем со свойством (10) является система $\mathrm{H}=\left(H_{1}, \ldots, H_{M} ; \pi_{1}, \ldots, \pi_{M}\right)$ таких подгрупп $H_{1}, \ldots, H_{M}$ конечной группы $G$ и подстановок $\pi_{1}, \ldots, \pi_{M}$, что $H_{r} \cap H_{s}=\{e\}, 1 \leqslant r<s \leqslant M$, где $e$ - единичный элемент группы $G$, и $\pi_{r}(e)=e, r=1, \ldots, M$.

Для системы Н удовлетворяющей условию (10), определим действующую на прямом произведении $H=H_{1} \times \ldots \times H_{M}$ подстановку $\pi=\pi_{1} \times \ldots \times \pi_{M}$ :

$$
\pi\left(a_{1}, a_{2}, \ldots, a_{M}\right)=\left(\pi_{1}\left(a_{1}\right), \pi_{2}\left(a_{2}\right), \ldots, \pi_{M}\left(a_{M}\right) .\right.
$$


Цикловую структуру $\operatorname{Str}\left(\pi_{1} \times \ldots \times \pi_{M}, H_{1} \times \ldots \times H_{M}\right)$ подстановки (11) для системы множеств $\mathrm{H}=\left(H_{1}, \ldots, H_{M} ; \pi_{1}, \ldots, \pi_{M}\right)$, удовлетворяющей условию (10), назовём композицией структур $\operatorname{Str}\left(\pi_{r}, H_{r}\right), r=1, \ldots, M$. Операцию композиции обозначим символом $\star$ :

$$
\star_{i=1}^{M} \operatorname{Str}\left(\pi_{i}, H_{i}\right)=\operatorname{Str}\left(\pi_{1} \times \pi_{2} \times \ldots \times \pi_{M}, H_{1} \times H_{2} \times \ldots \times H_{m}\right) .
$$

Операция $\star$ в (12) обладает, очевидно, свойствами коммутативности и ассоциативности.

Предложение 5. Пусть система множеств $u$ подстановок $\mathrm{H}=$ $\left(H_{1}, \ldots, H_{M} ; \pi_{1}, \ldots, \pi_{M}\right)$ удовлетворяет условию (10) и при этом каждая подстановка $\pi_{i}$ на $H_{i}$ состоит из одного фрагмента: $\pi_{i}=\left(l_{i}\right)^{k_{i}}, i=1, \ldots, M$. Тогда

$$
\operatorname{Str}\left(\pi_{1} \times \ldots \times \pi_{M}, H_{1} \times \ldots \times H_{m}\right)=\left(\operatorname{HOK}\left(l_{1}, \ldots, l_{M}\right)\right)^{\frac{k_{1} \ldots k_{M} l_{1} \ldots l_{M}}{\operatorname{HOK}\left(l_{1}, \ldots, l_{M}\right)}} .
$$

Действительно, каждый элемент $\left(a_{1}, \ldots, a_{M}\right) \in H_{1} \times \ldots \times H_{M}$ принадлежит, очевидно, циклу длины $\mathrm{HOK}\left(l_{1}, \ldots, l_{M}\right)$. Число элементов в $H_{1} \times \ldots \times H_{M}$ равно $k_{1} l_{1} k_{2} l_{2} \ldots k_{M} l_{M}$. Следовательно, рассматриваемая подстановка имеет в точности $\frac{k_{1} \ldots k_{M} l_{1} \ldots l_{M}}{\operatorname{HOK}\left(l_{1}, \ldots, l_{M}\right)}$ циклов длины $\operatorname{HOK}\left(l_{1}, \ldots, l_{M}\right)$.

Следствие 4. Пусть $\mathrm{H}=\left(H_{1}, \ldots, H_{M} ; \pi_{1}, \ldots, \pi_{M}\right)$ - система из множеств u подстановок, удовлетворяющая условию (10). Пусть, далее, $\operatorname{Str}\left(\pi_{r}, H_{r}\right)=$ $\prod_{j_{r}=1}^{t_{r}}\left(l_{r j_{r}}\right)^{k_{r j_{r}}}$ и $H_{r j_{r}} \subseteq H_{r}-$ подмножество, на котором действует фрагмент $\pi_{r j_{r}}=\left(l_{r j_{r}}\right)^{k_{r j_{r}}}, j_{r}=1, \ldots, t_{r}, r=1, \ldots, M$. Тогда:

а) для любого набора $\bar{j}=\left(j_{1}, \ldots, j_{M}\right), 1 \leqslant j_{r} \leqslant t_{r}, r=1, \ldots, M$, система $\mathrm{H}(\bar{j})=$ $\left(H_{1 j_{1}}, \ldots, H_{M j_{M}} ; \pi_{1 j_{1}}, \ldots, \pi_{M j_{M}}\right)$ удовлетворяет условию (10) и

$$
\begin{gathered}
\operatorname{Str}(\pi, \mathrm{H}(\bar{j}))=\star_{r=1}^{M} \operatorname{Str}\left(\pi_{r j_{r}}, H_{r j_{r}}\right)= \\
=\left(\operatorname{HOK}\left(l_{1 j_{1}}, \ldots, l_{M j_{M}}\right)\right)^{\frac{k_{1 j_{1} l_{1 j_{1}} \ldots k_{M j_{M}} l_{M j_{M}}}}{\operatorname{HOK}\left(l_{1 j_{1} \ldots l_{M j_{M}}}\right)},}
\end{gathered}
$$

б) для $H=H_{1} \times \ldots \times H_{M} u \pi=\pi_{1} \times \ldots \times \pi_{M}$ справедливо равенство

$$
\operatorname{Str}(\pi, H)=\prod_{\bar{j}}\left(\star_{r=1}^{M} \operatorname{Str}\left(\pi_{r j_{r}}, H_{r j_{r}}\right)\right),
$$

где произведение берется по всем $T=t_{1} t_{2} \ldots t_{M}$ наборам $\bar{j}$.

Доказательство. а) Всякая система Н $(\bar{j})$ удовлетворяет условию (10), так как этому условию удовлетворяет система Н. Поэтому для любого $\bar{j}$ из Предложения 5 следует, что $\star_{j=1}^{M} \operatorname{Str}\left(\pi_{r j_{r}}, H_{r j_{r}}\right)$ состоит из одного фрагмента, цикловая структура которого выражается формулой (13).

б) Для любого $h \in \prod_{r=1}^{M} H_{r}$ найдется такой вектор $\bar{j}=\left(j_{1}, \ldots, j_{M}\right)$, что $h \in H(\bar{j})$. Ясно также, что если $\bar{j} \neq \bar{j}^{\prime}$, то $H(\bar{j}) \cap H\left(\bar{j}^{\prime}\right)=\varnothing$. Поэтому $H$ разбивается на $T$ попарно не пересекающихся множеств $H(\bar{j})$. Пусть $(n)^{K}-$ произвольно взятый фрагмент в $\operatorname{Str}(\pi, H)$ и $H(n)$ - множество всех элементов $H$, принадлежащих циклам 
длины $n$; имеем $|H(n)|=K n$. Мы видели в п. а), что $\operatorname{Str}(\pi, H(\bar{j}))$ для любого $\bar{j}$ состоит из одного фрагмента (см. (13)). Если $h \in H(n)$, то $h$ входит в одно из множеств $H\left(\bar{j}_{1}\right), \ldots, H\left(\bar{j}_{x}\right)$, где $H\left(\bar{j}_{i}\right)$ при $i=1, \ldots, x$ - все множества $H(\bar{j})$ с цикловой структурой вида $(n)^{k}$, т. е. $\operatorname{Str}\left(\pi, H\left(\bar{j}_{i}\right)\right)=(n)^{k^{(i)}}, i=1, \ldots, x$. Сравнивая число элементов в $H(n)$ и во всех этих $H\left(\bar{j}_{i}\right), i=1, \ldots, x$, получаем: $K n=k^{(1)} n+\ldots+k^{(x)} n$, или $K=k^{(1)}+\ldots+k^{(x)}$. Таким образом, $(n)^{K}=\prod_{i=1}^{x}(n)^{k^{(i)}}$. Это доказывает справедливость утверждения б).

Мы будем далее применять Предложение 5 и Следствие 4 к случаю, когда $H=$ $H_{1} \times \ldots \times H_{M}$, где $H_{r}(r=1, \ldots, M)$ - примарные циклические группы, а $\pi_{r}-$ степенное отображение $f^{(d)}$, действующее на $H_{r}$ как подстановка.

\section{3. Цикловая структура степенных отображений на примарной циклической группе нечётного поряд-} Ka

Пусть сначала $u \geqslant 2$ - простое число, $\alpha \geqslant 1-$ целое, $C_{u^{\alpha}}=\langle g\rangle-$ примарная циклическая группа с единицей $e$, порождаемая элементом $g$. Для целых чисел $d \geqslant 2$, не делящихся на $u$, рассматриваем отображения $f^{(d)}$ на группе $C_{u^{\alpha}}: f^{(d)}(x)=x^{d}$. Поскольку $g^{u^{\alpha}}=e$, результат действия $f^{(d)}$ зависит фактически только от наименьшего неотрицательного вычета $\bar{d}$ числа $d$ по модулю $u^{\alpha}$. Поэтому далее мы часто вместо $f^{(d)}$ рассматриваем отображение $f^{(\bar{d})}$, ограничивая тем самым область значений $d$ отрезком $\left[1, u^{\alpha}-1\right]$. Отметим сразу частный случай, когда цикловая структура отображения определяется тривиально.

Предложение 6. Пусть $u \geqslant 2$ - простое число, $\alpha \geqslant 1-$ иелое. Тогда

$$
\operatorname{Str}\left(f^{(d)}, C_{u^{\alpha}}\right)=(1)^{u^{\alpha}}
$$

для любого $d \equiv 1\left(\bmod u^{\alpha}\right)$.

Ввиду Предложения 6 исключаем случай $d \equiv 1\left(\bmod u^{\alpha}\right)$ из дальнейшего рассмотрения - в этом параграфе и в $\$ 4$.

Каждое целое число из $\left[1, u^{\alpha}-1\right]$ можно записать как значение в точке $u$ многочлена степени не выше $\alpha-1$ с коэффициентами из $\{0,1, \ldots, u-1\}$ :

$$
d=t_{0}+t_{1} u+\ldots+t_{\alpha-1} u^{\alpha-1}, \quad t_{i} \in\{0,1, \ldots, u-1\}, 0 \leqslant i<\alpha .
$$

Таким образом, всякое $d$ можно представить элементом (15) кольца $\mathbb{Z}_{u^{\alpha}}$. Ясно, что в $(15) d \in \mathbb{Z}_{u^{\alpha}}^{*}$ тогда и только тогда, когда $t_{0} \neq 0$.

Далее в этом параграфе мы рассматриваем только нечётные простые $u$. Цикловая структура степенных отображений на примарной группе $C_{2^{\alpha}}$ будет описана в $\S 4$.

Мы видели в 11 (Предложения 2 и 3, Следствие 2), что при нечётном и группа $\operatorname{pm}\left(C_{u^{\alpha}}\right)$ и изоморфная ей группа $\mathbb{Z}_{u^{\alpha}}^{*}$ - циклические группы порядка $u^{\alpha}-u^{\alpha-1}$ и потому каждый элемент $d \in \mathbb{Z}_{u^{\alpha}}^{*}$ имеет порядок, являющийся делителем $(u-1) u^{\alpha-1}$.

Укажем некоторые нужные нам свойства элементов кольца $\mathbb{Z}_{u^{\alpha}}$. 
Лемма 1. Пусть $и$-нечётное простое, $\alpha \geqslant 1-$ иелое число, $d \geqslant 2$ - иелое число, $u \nmid d, d \neq \equiv 1\left(\bmod u^{\alpha}\right)$, представленное в виде $(12)$ :

$$
d=\sum_{i=0}^{\alpha-1} t_{i} u^{i}, \quad 0 \leqslant t_{i} \leqslant u-1, \quad i=0,1, \ldots, \alpha-1 .
$$

Порядок $d$ в группе $\mathbb{Z}_{u^{\alpha}}^{*}$ есть степень и в том и только в том случае, когда $t_{0}=1$. Если $t_{0}=1$, то порядок $d$ равен $u^{\alpha-\beta}$, где $\beta=\min _{1 \leqslant k \leqslant \alpha-1}\left\{k: t_{k} \neq 0\right\}$.

Действительно, при $t_{0} \neq 1$ элемент $d$ из (15) не может иметь порядок, равный степени $u$, по малой теореме Ферма. Если же $d=1+\sum_{i=\beta}^{\alpha-1} t_{i} u^{i}, t_{\beta} \neq 0$, то порядок $d$ в $\mathbb{Z}_{u^{\alpha}}^{*}$, очевидно, равен $u^{\alpha-\beta}$.

Для произвольного целого $d \geqslant 2$, не делящегося на нечётное простое $u$, вводим два параметра, которые, как будет показано в Предложении 7, полностью определяют цикловую структуру степенного отображения $f^{(d)}$ на циклической группе $C_{u^{\alpha}}, \alpha \geqslant 1$.

1) Обозначим через $\operatorname{ord}(d)$ порядок числа $d$ по модулю $u$ :

$$
\operatorname{ord}(d)=\operatorname{ord}(d, u)=\min \left\{k: d^{k} \equiv 1 \quad(\bmod u)\right\}
$$

$\left(\operatorname{ord}(d) \mid(u-1)\right.$ по малой теореме Ферма). Ясно, что $d^{\operatorname{ord}(d)}-1$ делится на некоторую степень $u^{r}$ числа $u, r \geqslant 1$.

2) Обозначим через $\rho$ наибольшую не превышающую $\alpha$ степень $u$, на которую делится $d^{\operatorname{ord}(d)}-1$ :

$$
\rho=\rho(d, u)=\max _{1 \leqslant \beta \leqslant \alpha}\left\{\beta: u^{\beta} \mid\left(d^{\operatorname{ord}(d)}-1\right)\right\} .
$$

Отметим, что для нахождения параметров $\operatorname{ord}(d)$ и $\rho$ не нужно находить представление $d$ в виде (15). Предложение 7 мы формулируем в предположении $2 \leqslant d \leqslant$ $u^{\alpha}-1$ лишь для удобства изложения.

Предложение 7. Пусть $и$ - нечётное простое число, $\alpha \geqslant 1$ - иелое число и для иелого числа $d$, где $2 \leqslant d \leqslant u^{\alpha}-1, u \nmid d$, рассматриваем степенное отображение $f^{(d)}: x \rightarrow x^{d}$ на грynnе $C_{u^{\alpha}}$. Тогда

$$
\operatorname{Str}\left(f^{(d)}, C_{u^{\alpha}}\right)=(1)(\operatorname{ord}(d))^{\frac{u^{\rho}-1}{\operatorname{ord}(d)}} \prod_{i=1}^{\alpha-\rho}\left(\operatorname{ord}(d) u^{i}\right)^{\frac{u-1}{\operatorname{ord}(d)} u^{\rho}-1},
$$

где ord (d), $\rho$ - величины из (16) и (17) соответственно.

Доказательство. Найдем порядок $d$ в группе $\mathbb{Z}_{u^{\alpha}}^{*}$. Порядок этой группы равен $(u-1) u^{\alpha-1}$. Поэтому порядок элемента $d$ есть число вида $v u^{\alpha-\beta}$, где $v \mid(u-1)$, $1 \leqslant \beta \leqslant \alpha$. Так как $(u, v)=1$, то порядок элемента $d^{v}$ есть либо 1 (при $\left.\beta=\alpha\right)$, либо положительная степень $u$. В первом случае $d^{v} \equiv 1\left(\bmod u^{\alpha}\right)$, и потому $v=\operatorname{ord}(d)$. Во втором, по лемме $1, d^{v} \equiv 1+\sum_{i=1}^{\alpha-1} t_{i} u^{i}$, и вновь $v=\operatorname{ord}(d)$. Тогда, по определению $\rho, d^{v}=d^{\operatorname{ord}(d)} \equiv 1+\sum_{i=\rho}^{\alpha-1} t_{i} u^{i}\left(\bmod u^{\alpha}\right), t_{\rho} \neq 0$. По лемме 1 порядок элемента в правой части сравнения есть $u^{\alpha-\rho}$, и мы получаем, что порядок $d$ в $\mathbb{Z}_{u^{\alpha}}$ есть $\operatorname{ord}(d) u^{\alpha-\rho}$.

Введём теперь некоторую классификацию элементов группы $C_{u^{\alpha}}$, не равных $e$. Всякий элемент представим в виде

$$
h=g^{s(u) u^{\beta}},
$$


где $s(u)=s_{\beta}+s_{\beta+1} u+\ldots+s_{\alpha-1} u^{\alpha-\beta-1}, s_{\beta}, s_{\beta+1}, \ldots, s_{\alpha-1} \in \mathbb{Z}_{u}, s_{\beta} \neq 0$, при некотором $\beta, 0 \leqslant \beta \leqslant \alpha-1$. Для $\beta=0,1, \ldots, \alpha-1$ обозначим через $S_{\beta}$ совокупность всех элементов, представимых в виде (19) при данном $\beta$. Определим, далее, разбиение $C_{u^{\alpha}} \backslash\{e\}$ на непересекающиеся множества:

$$
\text { при } \alpha-\rho>0: \quad C_{u^{\alpha}} \backslash\{e\}=S_{0} \cup S_{1} \cup \ldots \cup S_{\alpha-\rho-1} \cup \widetilde{S}_{\alpha-\rho},
$$

где $\widetilde{S}_{\alpha-\rho}=S_{\alpha-\rho} \cup S_{\alpha-\rho+1} \cup \ldots \cup S_{\alpha-1}$;

$$
\text { при } \alpha-\rho=0: C_{u^{\alpha}} \backslash\{e\}=\widetilde{S}_{0} .
$$

Число элементов в $S_{\beta}$ равно $\left|S_{\beta}\right|=u^{\alpha-\beta}-u^{\alpha-\beta-1}$; число элементов в $\widetilde{S}_{\alpha-\rho}$, как нетрудно заметить, равно $\left|\widetilde{S}_{\alpha-\rho}\right|=u^{\rho}-1$. Будем рассматривать действие $f^{(d)}$ на компонентах разбиения (20).

Найдем длины циклов, которым принадлежат элементы этих подмножеств. Ясно, что эти длины - делители $\operatorname{ord}(d) u^{\alpha-\rho}$, порядка элемента $d$ в группе $\mathbb{Z}_{u^{\alpha}}^{*}$. По определению $\rho$

$$
d^{\operatorname{ord}(d)} \equiv 1+t_{\rho} u^{\rho}+\sum_{i=\rho+1}^{\alpha-1} t_{i} u^{i} \quad\left(\bmod u^{\alpha}\right), \quad t_{i} \in \mathbb{Z}_{u}, \quad t_{\rho} \neq 0 .
$$

Рассмотрим два случая.

1) Пусть $\beta$ - одно из чисел $0,1, \ldots, \alpha-\rho-1$. Ввиду $(21)$

$$
u^{\beta} d^{\operatorname{ord}(d) u^{\gamma}} \equiv u^{\beta}+t_{\rho} u^{\beta+\rho+\gamma} \begin{cases}\equiv u^{\beta}\left(\bmod u^{\alpha}\right), & \gamma=\alpha-\rho-\beta, \\ \equiv u^{\beta}\left(\bmod u^{\alpha}\right), & \gamma<\alpha-\rho-\beta .\end{cases}
$$

Из (22) следует, что $g^{u^{\beta}}$ принадлежит циклу длины $\operatorname{ord}(d) u^{\alpha-\rho-\beta}$. Так как $(a, u)=1$, то любой элемент $h=g^{\alpha u^{\beta}} \in S_{\beta}$, тоже принадлежит некоторому циклу той же длины, и мы получаем, что при любом $\beta=0,1, \ldots, \alpha-\rho-1$ все элементы $S_{\beta}$ принадлежат циклам длины $\operatorname{ord}(d) u^{\alpha-\rho-\beta}$.

2) Пусть $h \in \widetilde{S}_{\alpha-\rho}$, т. е. $h=g^{a u^{\alpha-\rho+k}}$, где $(a, u)=1, k \geqslant 0$. Тогда ввиду $(21)$

$$
a u^{\alpha-\rho+k} d^{\operatorname{ord}(d)} \equiv a u^{\alpha-\rho+k}+a t_{\rho} u^{\rho+\alpha-\rho+k} \equiv a u^{\alpha-\rho+k} \quad\left(\bmod u^{\alpha}\right),
$$

и поэтому каждый элемент $\widetilde{S}_{\alpha-\rho}$ принадлежит циклу длины ord $(d)$. Таким образом, для $\beta=0,1, \ldots, \alpha-\rho-1$ все элементы $S_{\beta}$ распределяются по

$$
\frac{\left|S_{\beta}\right|}{\operatorname{ord}(d) u^{\alpha-\rho-\beta}}=\frac{u^{\alpha-\beta}-u^{\alpha-\beta-1}}{\operatorname{ord}(d) u^{\alpha-\rho-\beta}}=\frac{u-1}{\operatorname{ord}(d)} u^{\rho-1}
$$

циклам длины $\operatorname{ord}(d) u^{\alpha-\rho-\beta}$ :

$$
\operatorname{Str}\left(f^{(d)}, S_{\beta}\right)=\left(\operatorname{ord}(d) u^{\alpha-\rho-\beta}\right)^{\frac{u-1}{\operatorname{ord}(d)} u^{\rho-1}}, \quad \beta=0,1, \ldots, \alpha-\rho-1 .
$$

Все элементы $\widetilde{S}_{\alpha-\rho}$ входят в

$$
\frac{\left|\widetilde{S}_{\alpha-\rho}\right|}{\operatorname{ord}(d)}=\frac{u^{\rho}-1}{\operatorname{ord}(d)}
$$


циклов длины $\operatorname{ord}(d)$, и

$$
\operatorname{Str}\left(f^{(d)}, \widetilde{S}_{\alpha-\rho}\right)=\operatorname{ord}(d)^{\frac{u^{\rho}-1}{\operatorname{ord}(d)}}
$$

В последовательности цикловых структур

$$
\operatorname{Str}\left(f^{(d)}, \widetilde{S}_{\alpha-\rho}\right), \operatorname{Str}\left(f^{(d)}, \widetilde{S}_{\alpha-\rho-1}\right), \ldots, \operatorname{Str}\left(f^{(d)}, \widetilde{S}_{1}\right), \operatorname{Str}\left(f^{(d)}, \widetilde{S}_{0}\right)
$$

длины циклов в каждой из структур делятся на длины циклов во всех предшествующих. Поэтому $\operatorname{Str}\left(f^{(d)}, C_{u^{\alpha}}\right)$ получается объединением всех фрагментов в $(23)$, (24) и фрагмента (1) (соответствующего единице $e)$ :

$$
\operatorname{Str}\left(f^{(d)}, C_{u^{\alpha}}\right)=(1)\left(\operatorname{ord}(d)^{\frac{u^{\rho}-1}{\operatorname{ord}(d)}} \prod_{i=1}^{\alpha-\rho}\left(\operatorname{ord}(d) u^{i}\right)\right)^{\frac{u-1}{\operatorname{ord}(d)} u^{\rho-1}},
$$

т. е. справедлива формула (18).

Предложение доказано.

Если мы произвольно берём натуральное число $d$, то, при любом нечётном простом $u$ с вероятностью $1-\frac{1}{u}$ это будет число с $\rho(d, u)=1$. В этом случае формула (18) упрощается.

Следствие 5. Пусть и-нечётное простое, $\alpha \geqslant 1$ - иелое число. Если $d \geqslant 2$, $u \nmid d, \rho(d, u)=1$, mo

$$
\operatorname{Str}\left(f^{(d)}, C_{u^{\alpha}}\right)=(1) \prod_{i=0}^{\alpha-1}\left(\operatorname{ord}(d) u^{i}\right)^{\frac{u-1}{\operatorname{ord}(d)}}
$$

в частности,

$$
\operatorname{Str}\left(f^{(d)}, C_{u}\right)=(1)(\operatorname{ord}(d))^{\frac{u-1}{\operatorname{ord}(d)}}
$$

\section{4. Цикловая структура степенного отображения на примарной циклической 2-группе}

В этом параграфе мы найдём формулы для цикловой структуры степенного отображения $f^{(d)}$ (где $d-$ произвольное целое нечётное число) на группе $C_{2^{\alpha}}, \alpha \geqslant 1-$ любое целое число. Вначале отметим несколько частных случаев.

Предложение 8. а) Для любого нечётного $d$

$$
\operatorname{Str}\left(f^{(d)}, C_{2}\right)=(1)^{2} ;
$$

б) для любого нечётного $d \geqslant 3$

$$
\operatorname{Str}\left(f^{(d)}, C_{4}\right)= \begin{cases}(1)^{4}, & d \equiv 1(\bmod 4) \\ (1)^{2}(2), & d \equiv-1(\bmod 4) .\end{cases}
$$

Утверждения очевидны. 
Предложение 9. Пусть $C_{2^{\alpha}}=\langle g\rangle, \quad e-$ единица группь $C_{2^{\alpha}}$. Тогда

$$
\operatorname{Str}\left(f^{\left(2^{\alpha}-1\right)}, C_{2^{\alpha}}\right)=(1)^{2}(2)^{2^{\alpha-1}-1} .
$$

Действительно, для любого целого $а$ и $d=2^{\alpha}-1$ справедливо равенство $g^{a d}=$ $g^{-a}$, и поэтому имеется 2 цикла длины $1:(e),\left(g^{2^{\alpha-1}}\right)$ и $2^{\alpha-1}-1$ циклов длины 2 : $\left(g^{k}, g^{-k}\right), k=1,2, \ldots, 2^{\alpha-1}-1$.

Учитывая Предложения 8, 9 и Предложение 6 в § 3, мы далее здесь всегда предполагаем, что $\alpha \geqslant 3$ и $3 \leqslant d<2^{\alpha}-2$. Найдём, прежде всего, порядок $d$ в группе $\mathbb{Z}_{2^{\alpha}}^{*}$. Следствие 3 в $\S 2$ показывает, что он есть степень 2 и не превосходит $2^{\alpha-2}$. Укажем конкретный способ его нахождения. Для нечётного $d$ и целого числа $\alpha \geqslant 3$ при $3 \leqslant d<2^{\alpha}$ определяем целочисленный параметр $v$ :

$$
\begin{gathered}
v=v(d, \alpha)= \\
=\max \left\{k \in\{2, \ldots \alpha-1\}:\left\{\begin{array}{ll}
2^{k} \mid(d-1), & d \equiv 1(\bmod 4), \\
2^{k} \mid(d+1), & d \equiv-1(\bmod 4)
\end{array}\right\} .\right.
\end{gathered}
$$

Лемма 2. Пусть $\alpha \geqslant 3$ - целое число, $d$ - нечётное иелое из множества $\left\{3, \ldots, 2^{\alpha}-3\right\}$. Тогда порядок $d$ в группе $\mathbb{Z}_{2^{\alpha}}^{*}$ равен $2^{\alpha-v}$, где $v-$ число из (25).

Доказательство. Пусть $d \equiv 1(\bmod 4)$. Тогда $d-1$ делится на $2^{v}$ и $d=1+2^{v} n$, где $n$ - нечётное число. Таким образом,

$$
d \equiv 1+2^{v} n\left(\bmod 2^{v+1}\right) .
$$

Отсюда получаем:

$$
d^{2^{k}} \equiv 1+2^{v+k} n\left(\bmod 2^{v+k+1}\right), \quad n \text { нечётно, } k=1,2, \ldots,
$$

и $d^{2^{\alpha-v}} \equiv 1\left(\bmod 2^{\alpha}\right)$ при $k=\alpha-v$; очевидно, что $d^{2^{k}} \not \equiv 1\left(\bmod 2^{\alpha}\right)$, если $k<\alpha-v$.

Пусть теперь $d \equiv-1(\bmod 4)$. Имеем тогда

$$
d \equiv-1+2^{v} n\left(\bmod 2^{v+1}\right), \quad n \text { нечётно. }
$$

Из (27) получается:

$$
d^{2^{k}} \equiv 1-2^{v+k} n\left(\bmod 2^{v+k+1}\right), \quad n \text { нечётно, } k=1,2, \ldots
$$

Отсюда снова следует, что порядок $d$ есть $2^{\alpha-v}$. Лемма доказана.

Отметим некоторые сравнения, следующие из (26) и, соответственно, (27), (28).

Если $\alpha \geqslant 3, d \in\left\{3, \ldots, 2^{\alpha}-3\right\}$ и $d \equiv 1(\bmod 4)$, то

$$
2^{\beta} d^{2^{\alpha-v-\gamma}} \equiv 2^{\beta}+2^{\alpha-\gamma+\beta}\left(\bmod 2^{\alpha-\gamma+\beta+1}\right),
$$

для целых $\beta, \gamma, \beta \geqslant 0,0 \leqslant \gamma \leqslant \alpha-v-1$. 
Если $\alpha \geqslant 3, d \in\left\{3, \ldots, 2^{\alpha}-3\right\}, d \equiv-1(\bmod 4)$, то

$$
2^{\beta} d \equiv-2^{\beta}+2^{v+\beta}\left(\bmod 2^{v+\beta+1}\right)
$$

и

$$
2^{\beta} d^{2^{\alpha-v-\gamma}} \equiv 2^{\beta}-2^{\alpha-\gamma+\beta}\left(\bmod 2^{\alpha-\gamma+\beta+1}\right),
$$

для целых $\beta, \gamma \geqslant 0,0 \leqslant \gamma \leqslant \alpha-v-1$.

Введём, аналогично $\S 3$, классификацию элементов $C_{2^{\alpha}}$. Пусть для $\beta=0,1, \ldots, \alpha-1$

а также

$$
S_{\beta}=\left\{g^{a 2^{\beta}}, \text { а нечётно }\right\},
$$

$$
\widetilde{S}_{\alpha-\beta}=S_{\alpha-\beta} \cup S_{\alpha-\beta+1} \cup \ldots \cup S_{\alpha-1}, \quad \beta=1, \ldots, \alpha-1 .
$$

Ясно, что $S_{\beta} \cap S_{\beta^{\prime}}=\varnothing$, если $\beta^{\prime} \neq \beta$. Нетрудно также показать, что

$$
\left|S_{\beta}\right|=2^{\alpha-\beta-1}, \quad \beta=0,1, \ldots, \alpha-1,
$$

И

$$
\left|\widetilde{S}_{\alpha-\beta}\right|=2^{\beta}-1 \text {. }
$$

Предложение 10. Пусть $\alpha \geqslant 3$ - целое, $d$ - нечётное иелое число из множества $\left\{3, \ldots, 2^{\alpha}-3\right\}$. Тогда

$$
\operatorname{Str}\left(f^{(d)}, C_{2^{\alpha}}\right)= \begin{cases}(1)^{2^{v}} \prod_{i=v}^{\alpha-1}\left(2^{\alpha-i}\right)^{2^{v-1}}, & d \equiv 1(\bmod 4), \\ (1)^{2}(2)^{2^{v}-1} \prod_{i=v}^{\alpha-2}\left(2^{\alpha-i}\right)^{2^{v-1}}, & d \equiv-1(\bmod 4),\end{cases}
$$

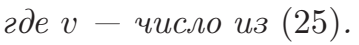

Доказательство. Рассмотрим разбиение:

$$
C_{2^{\alpha} \backslash\{e\}}=S_{0} \cup S_{1} \cup \ldots \cup S_{\alpha-v-1} \cup \widetilde{S}_{\alpha-v},
$$

где $S_{\beta}(\beta=0,1, \ldots, \alpha-v-1), \widetilde{S}_{\alpha-v}-$ множества, соответственно, из (32) и (33). Найдем сначала $\operatorname{Str}\left(f^{(d)}, S_{\beta}\right), \beta=0,1, \ldots, \alpha-v-1$. Для этого заметим, что длина цикла, которому принадлежит каждый из элементов $h=g^{2^{\beta} a} \in S_{\beta}$ (a нечётно), равна $2^{\alpha-v-\beta}$.

Действительно, $2^{\beta} d^{\alpha-v-\gamma}=2^{\beta} \pm 2^{\alpha-\gamma+\beta}\left(\bmod 2^{\alpha-\gamma+\beta+1}\right)$ согласно $(29)$ и $(31)$, и потому $a 2^{\beta} d^{\alpha-v-\gamma} \equiv a 2^{\beta}\left(\bmod 2^{\alpha}\right)$ тогда и только тогда, когда $\gamma \leqslant \beta$. Следовательно, длина цикла, которому принадлежит всякий элемент $h \in S_{\beta}$, на самом деле равна $2^{\alpha-v-\beta}$. Так как $\left|S_{\beta}\right|=2^{\alpha-\beta-1}$ по $(34)$, то число таких циклов равно $\frac{2^{\alpha-\beta-1}}{2^{\alpha-v-\beta}}=2^{v-1}$ и

$$
\operatorname{Str}\left(f^{(d)}, S_{\beta}\right)=\left(2^{\alpha-v-\beta}\right)^{2^{v-1}}, \quad \beta=0,1, \ldots, \alpha-v-1 .
$$

Рассмотрим далее действие $f^{(d)}$ на $\widetilde{S}_{\alpha-v}$. Если $d \equiv 1(\bmod 4)$, то непосредственно проверяется, что $h^{d} \equiv h\left(\bmod 2^{\alpha}\right)$ для любого $h \in \widetilde{S}_{\alpha-v}$. Так как $\left|\widetilde{S}_{\alpha-v}\right|=2^{v}-1$ (см. (35)), то

$$
\operatorname{Str}\left(f^{(d)}, \widetilde{S}_{\alpha-v}\right)=(1)^{2^{v}-1} \text {, если } d \equiv 1(\bmod 4) .
$$


Пусть теперь $d \equiv-1(\bmod 4)$. Сравнение $(30)$ показывает, что $2^{\beta} d \equiv-2^{\beta}\left(\bmod 2^{\alpha}\right)$ для всех $\beta \geqslant \alpha-v$, и потому $h^{d}=h^{-1}$, если $h \in \widetilde{S}_{\alpha-v}$. Таким образом (учитывая, что $\left|\widetilde{S}_{\alpha-v}\right|=2^{v}-1$ ), мы получаем, что при действии $f^{(d)}, d \equiv-1(\bmod 4)$, на $\widetilde{S}_{\alpha-v}$ имеется один цикл $\left(g^{2^{\alpha-1}}\right)$ длины 1 и $\frac{1}{2}\left(2^{v}-2\right)=2^{v-1}-1$ циклов $\left(g^{a d}, g^{-a}\right), a=1,2, \ldots, 2^{v-1}-1$, длины 2 , т. е.

$$
\operatorname{Str}\left(f^{(d)}, \widetilde{S}_{\alpha-v}\right)=(1)(2)^{2^{v-1}-1}, \text { если } d \equiv-1(\bmod 4) .
$$

Из $(37)$ и $(39)$ следует, что при $d \equiv-1(\bmod 4)$ циклы длины 2 возникают в результате действия $f^{(d)}$ на два подмножества в разбиении (36): на $S_{\alpha-v-1}$ и на $\widetilde{S}_{\alpha-v}$. Поэтому общее число циклов длины 2 при $d \equiv-1(\bmod 4)$ есть $2^{v-1}+2^{v-1}-1=2^{v}-1$.

Заметим теперь, что в последовательности цикловых структур

$$
\operatorname{Str}\left(f^{(d)}, \widetilde{S}_{\alpha-v}\right), \operatorname{Str}\left(f^{(d)}, S_{\alpha-v-1}\right), \ldots, \operatorname{Str}\left(f^{(d)}, S_{1}\right), \operatorname{Str}\left(f^{(d)}, S_{0}\right)
$$

длины циклов в каждой из структур делятся на длины циклов всех предшествующих. Поэтому из (36)-(38) и, соответственно, (36), (37) и (39) следует, что

$$
\operatorname{Str}\left(f^{(d)}, C_{2^{\alpha}}\right)=(1)^{2^{v}} \prod_{i=v}^{\alpha-1}\left(2^{\alpha-i}\right)^{2^{v-1}}, \text { если } d \equiv 1(\bmod 4),
$$

и

$$
\operatorname{Str}\left(f^{(d)}, C_{2^{\alpha}}\right)=(1)^{2}(2)^{2^{v}-1} \prod_{i=v}^{\alpha-2}\left(2^{\alpha-i}\right)^{2^{v-1}} \text {, если } d \equiv-1(\bmod 4),
$$

где $v$ - число из $(25)(2 \leqslant v \leqslant \alpha-1)$.

Предложение доказано.

\section{5. Цикловая структура степенного отображения $f^{(d)}$ на $\mathbb{Z}_{N}^{*}$ при произвольных $d$ и $N$}

Мы можем теперь найти цикловую структуру отображения $f^{(d)}$ на множестве $\mathbb{Z}_{N}^{*}$ обратимых элементов кольца $\mathbb{Z}_{N}$ для произвольных $d$ и $N$. Как мы видели в $\S 1$, она может быть определена как цикловая структура подстановки, индуцируемой $f^{(d)}$ на максимальном подмножестве $\operatorname{Ker}\left(f^{(d)}, \mathbb{Z}_{N}^{*}\right) \subseteq \mathbb{Z}_{N}^{*}$, для которого данное отображение является взаимно однозначным. В Предложении 4 было описано алгебраическое строение $\operatorname{Ker}\left(f^{(d)}, \mathbb{Z}_{N}^{*}\right)$. Пусть

$$
N=2^{\nu_{0}} \prod_{i=1}^{m} p_{i}^{\nu_{i}}
$$

- каноническое разложение $N\left(\nu_{0}=0\right.$, если $N$ нечётно), пусть $L_{i}=p_{i}^{\nu_{i}}-p_{i}^{\nu_{i}-1}$ и при $L_{i}>1$

$$
L_{i}=\prod_{u \mid L_{i}} u^{\alpha(u, i)}, \quad i=0,1, \ldots, m,
$$


- каноническое разложение числа $L_{i}$. В каждом таком разложении $u$ пробегает все различные простые делители $L_{i}$, а $\alpha(u, i)$ - максимальный показатель степени $u$, на которую делится $L_{i}$; числа $u^{\alpha(u, i)}$ называем примарными компонентами в разложениях (41). Напомним (см. Предложение В), что

$$
\mathbb{Z}_{N}^{*} \cong \mathbb{Z}_{2^{\nu_{0}}}^{*} \times \prod_{i=1}^{m} \mathbb{Z}_{p_{i}^{\nu_{i}}}^{*}
$$

Кроме того (см. п. 1.2),

$$
\begin{gathered}
\mathbb{Z}_{2^{\nu_{0}}}^{*} \cong C_{2} \times C_{2^{\nu_{0}-2}} \quad \text { при } \nu_{0} \geqslant 2, \quad \mathbb{Z}_{2}^{*} \cong C_{1}, \\
\mathbb{Z}_{p_{i}^{\nu_{i}}}^{*} \cong C_{L_{i}} \cong \prod_{u \mid L_{i}} C_{u^{\alpha(u, i)}} .
\end{gathered}
$$

При $i=1, \ldots, m$ обозначим через $U_{i}=U_{i}(d)=\left(u_{i 1}, u_{i 2}, \ldots, u_{i s_{i}}\right)$ множество всех различных простых делителей $L_{i}$, взаимно простых с $d$, а через $\widetilde{U}_{i}=\widetilde{U}_{i}(d)=$ $\left(u_{i 1}^{\alpha_{i 1}}, u_{i 2}^{\alpha_{i 2}}, \ldots, u_{i s_{i}}^{\alpha_{i s_{i}}}\right)$ - множество соответствующих примарных компонент в $(41)$ (если $s_{i}=0$, то $U_{i}=\widetilde{U}_{i}=\varnothing$ ). Пусть также при нечетном $d$

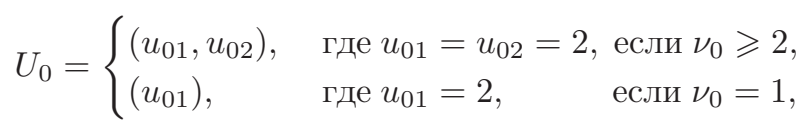

и

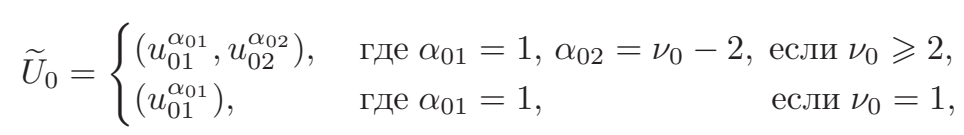

а при четном $d$ положим $U_{0}=\widetilde{U}_{0}=\varnothing$. Заменим двойные индексы в наборах $U=$ $\left(U_{0}, U_{1}, \ldots, U_{m}\right), \widetilde{U}=\left(\widetilde{U}_{0}, \widetilde{U}_{1}, \ldots, \widetilde{U}_{m}\right)$ номера от 1 до $M$ :

$$
U=\left(u_{1}, u_{2}, \ldots, u_{M}\right), \quad \widetilde{U}=\left(u_{1}^{\alpha_{1}}, u_{2}^{\alpha_{2}}, \ldots, u_{M}^{\alpha_{M}}\right) .
$$

В этих обозначениях формулу (7) в Предложении 4 из $§ 1$ можно записать в виде

$$
\operatorname{Ker}\left(f^{(d)}, \mathbb{Z}_{N}^{*}\right) \cong \prod_{r=1}^{M} C_{u_{r}^{\alpha_{r}}}
$$

здесь $M= \begin{cases}s_{1}+\ldots+s_{m} & \text { при } \nu_{0}=0, \\ 1+s_{1}+\ldots+s_{m} & \text { при } \nu_{0}=1, \text { Далее, будем иметь в виду представление } \\ 2+s_{1}+\ldots+s_{m} & \text { при } \nu_{0} \geqslant 2 .\end{cases}$ $\operatorname{Ker}\left(f^{(d)}, \mathbb{Z}_{N}^{*}\right)$ в виде $(43)$.

Введем обозначения $H=\operatorname{Ker}\left(f^{(d)}, \mathbb{Z}_{N}^{*}\right), H_{r}=C_{u_{r}^{\alpha_{r}}}$. Изоморфизм в формуле (43) означает соответствующее китайской теореме об остатках взаимно однозначное соответствие

$$
z \Leftrightarrow\left(h_{1}, h_{2}, \ldots, h_{M}\right),
$$

где $z \in \operatorname{Ker}\left(f^{(d)}, \mathbb{Z}_{N}^{*}\right)$, а $h_{r}$ (при каждом $\left.r=1, \ldots, M\right)$ - элемент кольца $\mathbb{Z}_{N}$, принадлежащий циклической подгруппе $C_{u_{r}^{\alpha_{r}}}$ группы $\mathbb{Z}_{N}^{*} ;$ при этом $u_{r}^{\alpha_{r}}-$ примарная компонента разложения (41) для некоторого числа $L_{i}, i \in\{0,1, \ldots, m\}$. Ясно, что 
$1 \Leftrightarrow(1,1, \ldots, 1)$. Так как $C_{u_{r}^{\alpha}} \cap C_{u_{s}^{\alpha}}=\{1\}$ при $1 \leqslant r<s \leqslant M$ и $1^{d}=1$, то система $\mathrm{H}_{\pi}=\left(C_{u_{1}^{\alpha_{1}}}, C_{u_{2}^{\alpha_{2}}}, \ldots, C_{u_{M}^{\alpha_{M}}} ; \pi_{1}, \ldots, \pi_{M}\right)$, где подстановки $\pi_{r}$ соответствуют отображениям $h \rightarrow h^{d}, h \in H_{r}(r=1, \ldots, M)$, удовлетворяет условиям (10) из $\S 2$. Соответствующая подстановка $\pi=\pi_{1} \times \ldots \times \pi_{M}$ - это отображение $f^{(d)}: z \rightarrow z^{d}$ для любого $z \in \operatorname{Ker}\left(f^{(d)}, \mathbb{Z}_{N}^{*}\right)=\mathbb{Z}_{N}^{*}(d)$. Поэтому можно использовать результаты $\S 2$ для нахождения $\operatorname{Str}\left(f^{(d)}, \mathbb{Z}_{N}^{*}\right)=\operatorname{Str}(\pi, H)=\operatorname{Str}\left(f^{(d)}, H_{1} \times \ldots \times H_{M}\right)$ по цикловым структурам $\operatorname{Str}\left(f^{(d)}, C_{u_{r} \alpha_{r}}\right), r=1, \ldots, M$, описанным в $\S \S 3-4$. Если $u_{r}$ нечетно, то согласно Предложению 7

$$
\operatorname{Str}\left(\pi_{r}, H_{r}\right)= \begin{cases}(1)\left(a_{r}\right)^{\left(u_{r}^{\alpha_{r}}-1\right) / a_{r}} \prod_{j=1}^{\alpha_{r}-\rho_{r}}\left(a_{r} u_{r}^{j}\right)^{\left(u_{r}-1\right) u_{r}^{\rho_{r}-1} / a_{r}}, & d \neq 1\left(\bmod u_{r}^{\alpha_{r}}\right), \\ (1)^{u_{r}^{\alpha_{r}}}, & d \equiv 1\left(\bmod u_{r}^{\alpha_{r}}\right),\end{cases}
$$

где $a_{r}=\operatorname{ord}\left(d, u_{r}\right), \rho_{r}=\rho\left(d, u_{r}\right)$ - число из $(17) ;$ если $\alpha_{r}=1$ и, следовательно, $\rho_{r}=1$, то все фрагменты в (44), кроме первых двух, отсутствуют.

Для упрощения формул будем обозначать величину $v\left(\bar{d}, \alpha_{r}\right)$ из $(25)$ через $v_{r}(\bar{d}$ - наименьший неотрицательный вычет $d$ по модулю $\left.2^{\alpha_{r}}\right)$. Если $u_{r}=2$, то при $\alpha_{r} \geqslant 3$ согласно Предложениям 9 и 10

$$
\operatorname{Str}\left(\pi_{r}, H_{r}\right)= \begin{cases}(1)^{2^{v_{r}}}(2)^{2^{v_{r}-1}} \prod_{j=v_{r}}^{\alpha_{r}-2}\left(2^{\alpha_{r}-j}\right)^{2^{v_{r}-1}}, & d \equiv 1(\bmod 4), \\ (1)^{2}(2)^{2^{v_{r}-1}} \prod_{j=v_{r}}^{\alpha_{r}-2}\left(2^{\alpha_{r}-j}\right)^{2^{v_{r}-1}}, & d \equiv 1\left(\bmod 2^{a_{r}}\right), \\ (1)^{2^{\alpha_{r}}}, & d \equiv 1(\bmod 4),\end{cases}
$$

а при $\alpha_{r}=1$ или 2 , согласно Предложению 8, соответственно,

$$
\operatorname{Str}\left(\pi_{r}, H_{r}\right)=(1)^{2} \quad \text { и } \operatorname{Str}\left(\pi_{r}, H_{r}\right)= \begin{cases}(1)^{4}, & d \equiv 1(\bmod 4), \\ (1)^{2}(2), & d \equiv-1(\bmod 4) ;\end{cases}
$$

если же $v_{r}>\alpha_{r}-2$, т. е. $v_{r}=\alpha_{r}-1$, то все фрагменты в (45), помимо первых двух, отсутствуют.

Далее, каждое из множеств $H_{r}, r=1, \ldots, M$, разбивается на непересекающиеся подмножества $H_{r j_{r}}$ так, что на $H_{r j_{r}}$ подстановка $\pi_{r}$ действует как какой-либо один фрагмент из структур в $(44),(45)$ или (46). А именно, при $d \not \equiv 1\left(\bmod u_{r}^{\alpha_{r}}\right)$ :

$$
\begin{gathered}
\operatorname{Str}\left(\pi_{r}, H_{r j_{r}}\right)= \begin{cases}\left(a_{r} u_{r}^{j_{r}}\right)^{\frac{1}{a_{r}}\left(u_{r}-1\right) u_{r}^{\rho_{r}-1}}, & u_{r} \text { нечётно, } 1 \leqslant j_{r} \leqslant \alpha_{r}-\rho_{r}, \\
\left(2^{j_{r}}\right)^{2^{v_{r}-1}}, & u_{r}=2, \alpha_{r} \geqslant 3,2 \leqslant j_{r} \leqslant \alpha_{r}-v_{r} ;\end{cases} \\
\operatorname{Str}\left(\pi_{r}, H_{r, 0}\right)=\left(a_{r}\right)^{\frac{1}{a_{r}}\left(u_{r}^{\rho_{r}}-1\right)}, \\
\operatorname{Str}\left(\pi_{r}, H_{r, 1}\right)= \begin{cases}(2)^{2^{v_{r}}-1}, & u_{r}=2, \alpha_{r} \geqslant 3, d \equiv 1(\bmod 4), \\
(2)^{2^{v_{r}}-1}, & u_{r}=2, \alpha_{r} \geqslant 3, \\
(2), & u_{r}=2, \alpha_{r}=2, d \equiv-1(\bmod 4) ;\end{cases} \\
\operatorname{Str}\left(\pi_{r}, H_{r,-1}\right)= \begin{cases}(1), & u_{r} \text { нечётно, } \\
(1)^{2^{v_{r}}}, & u_{r}=2, \alpha_{r} \geqslant 2, d \equiv(\bmod 4), \\
(1)^{2}, & u_{r}=2, \alpha_{r} \geqslant 2, d \equiv-1(\bmod 4) .\end{cases}
\end{gathered}
$$


Кроме того, пусть $H_{r,-1}=C_{u_{r}^{a_{r}}}$, если $d \equiv 1\left(\bmod u_{r}^{d_{r}}\right)$.

Предложение 11. Пусть $d \geqslant 2, N \geqslant 2$ - любое натуралъное число, представленное в виде $(40), \operatorname{Ker}\left(f^{(d)}, \mathbb{Z}_{N}^{*}\right) \cong \prod_{r=1}^{M} C_{u_{r}^{\alpha_{r}}}-$ представление $(43)$ для набора $U(d, N)$ простьх чисел в (42). Пусть, далее, $a_{r}=\operatorname{ord}\left(d, u_{r}\right), \rho_{r}=\rho\left(d, u_{r}\right)$ - число в (17) $u$ $v_{r}=v\left(\bar{d}, \alpha_{r}\right)$ - число в $(25)$, где $\bar{d}-$ наименъший неотрицательный вычет $d$ nо модулю $2^{\alpha_{r}}$.

а) Число $n$ является длиной цикла в $\operatorname{Str}\left(f^{(d)}, \mathbb{Z}_{N}^{*}\right)$ тогда и только тогда, когда

$$
n=\operatorname{HOK}\left(l_{1}, l_{2}, \ldots, l_{M}\right)
$$

где $l_{r} \in\left\{1, a_{r}, a_{r} u_{r}, \ldots, a_{r} u_{r}^{\alpha_{r}-\rho_{r}}\right\}$ при нечётном $u_{r}$, а если $u_{r}=2$, то

$$
l_{r} \in \begin{cases}\left\{1,2, \ldots, 2^{\alpha_{r}-v_{r}}\right\}, & \text { если } \alpha_{r} \geqslant 3 \\ \{1,2\}, & \text { если } \alpha_{r}=2, d \equiv-1(\bmod 4)\end{cases}
$$

u $l_{r}=1$, если $u_{2}=2$ и при этом либо $\alpha_{r}=2, d \equiv 1(\bmod 4)$, либо $\alpha_{1}=\ldots=$ $\alpha_{M}=1$.

б) Пусть $n-$ число из (50). Тогда общее число ииклов длины $n$ в $\operatorname{Str}\left(f^{(d)}, \mathbb{Z}_{N}^{*}\right)$ равно

$$
\frac{1}{n} \sum \prod_{r=1}^{M} b_{r}
$$

где $b_{r}=u_{r}^{\alpha_{r}} n p u d \equiv 1\left(\bmod u_{r}^{\alpha_{r}}\right)$, a если $d \not \equiv 1\left(\bmod u_{r}^{\alpha_{r}}\right)$, mo:

1) при нечётном $u_{r}$

$$
b_{r}= \begin{cases}1, & \text { если } l_{r}=1, \\ u_{r}^{\rho_{r}}-1, & \text { если } l_{r}=a_{r}, \\ \left(u_{r}-1\right) u_{r}^{j_{r}+\rho_{r}-1}, & \text { если } l_{r}=a_{r} u_{r}^{j_{r}}, 1 \leqslant j_{r} \leqslant \alpha_{r}-\rho_{r} ;\end{cases}
$$

2) $n p u u_{r}=2 u d \equiv 1(\bmod 4)$

$$
b_{r}= \begin{cases}2^{v_{r}}, & \text { если } l_{r}=1, \\ 2^{j_{r}+v_{r}-1}, & \text { если } l_{r}=2^{j_{r}}, 1 \leqslant j_{r} \leqslant \alpha_{r}-v_{r}\end{cases}
$$

3) прu $u_{r}=2 u d \equiv-1(\bmod 4)$

$$
b_{r}= \begin{cases}2, & \text { если } l_{r}=1 \text { или } l_{r}=2, \alpha_{r}=2, \\ 2\left(2^{v_{r}}-1\right), & \text { если } l_{r}=2, \alpha_{r} \geqslant 3, \\ 2^{j_{r}+v_{r}-1}, & \text { если } l_{r}=2^{j_{r}}, \alpha_{r}>3,2 \leqslant j_{r} \leqslant \alpha_{r}-v_{r}\end{cases}
$$

суммирование в (51) производится по всем возможным представлениям $n$ в виде (50). 
Доказательство. а) Исходя из набора $U(d, N)$ в $(42)$, состоящего из простых чисел, не делящих $d$, и представления $\operatorname{Ker}\left(f^{(d)}, \mathbb{Z}_{N}^{*}\right)=H$ в виде прямого произведения (43), рассмотрим действие отображения $f^{(d)}$ на введённых выше множествах $H_{1}, \ldots, H_{M}$, каждое из которых соответствует числу $u_{r} \in U(d, N)$. Положим при нечетном $u_{r}$

$$
I_{r}= \begin{cases}\{-1\}, & \text { если } d \equiv 1\left(\bmod u_{r}^{\alpha_{r}}\right), \\ \left\{-1,0,1, \ldots, \alpha_{r}-\rho_{r}\right\}, & \text { если } d \neq \equiv 1\left(\bmod u_{r}^{\alpha_{r}}\right), u_{r} \text { нечетно, }\end{cases}
$$

а при $u_{r}=2$

$$
I_{r}^{(0)}=\left\{\begin{array}{l}
\{-1\}, \quad \text { если } d \equiv 1\left(\bmod u_{r}^{\alpha_{r}}\right), \\
\left\{-1,1,2, \ldots, \alpha_{r}-v_{r}\right\}, \quad \text { если } d \neq 1\left(\bmod u_{r}^{\alpha_{r}}\right), u_{r}=2, \alpha_{r} \geqslant 3, \\
\{-1,1\}, \quad \text { если } d \not \equiv 1\left(\bmod u_{r}^{\alpha_{r}}\right), d \equiv-1(\bmod 4), u_{r}=\alpha_{r}=2 .
\end{array}\right.
$$

Возьмём произвольно последовательность $j_{1}, j_{2}, \ldots, j_{M}$, где $j_{r} \in I_{r}$, если $u_{r}$ нечётно, и $j_{r} \in I_{r}^{(0)}$ при $u_{r}=2$. Ей соответствует прямое произведение

$$
H_{1 j_{1}} \times H_{2 j_{2}} \times \ldots \times H_{M j_{M}}
$$

подмножеств множеств $H_{r}, r=1, \ldots, M$; как отмечено выше, на $H_{1} \times \ldots \times H_{M}$ и, следовательно, на (52), определена подстановка $\pi=\pi_{1} \times \ldots \times \pi_{M}$, где $\pi_{r}$ - подстановка на $H_{r}$, индуцируемая отображением $f^{(d)}$. Цикловая структура для каждого $H_{r j_{r}}$ в $(52)$ состоит из одного фрагмента, поэтому, согласно Следствию 4, $\operatorname{Str}\left(\pi, H_{1 j_{1}} \times \ldots \times H_{M j_{M}}\right)$ также состоит из одного фрагмента, и длина цикла в ней есть

$$
n=\operatorname{HOK}\left(l_{1}, l_{2}, \ldots, l_{M}\right), \text { где } l_{r}= \begin{cases}1, & j_{r}=-1, \\ a_{r} u_{r}^{j_{r}}, & j_{r} \in I_{r} \backslash\{-1\}, u_{r} \text { нечетное }, \\ 2^{j_{r}}, & j_{r} \in I_{r} \backslash\{-1\}, u_{r}=2 .\end{cases}
$$

Отсюда следует, что $l_{r}$ может принимать всего $t_{r}$ различных значений, где при $d \not \equiv 1$ $\left(\bmod u_{r}^{\alpha_{r}}\right)$

$$
t_{r}= \begin{cases}\alpha_{r}-\rho_{r}+2, & \text { если } u_{r} \text { нечетно, } \\ \alpha_{r}-v_{r}+1, & \text { если } u_{r}=2, \alpha_{2} \geqslant 3, \\ 2, & \text { если } u_{r}=2, \alpha_{r}=2, d \equiv-1 \quad(\bmod 4),\end{cases}
$$

и $t_{r}=1$ при $d \equiv 1\left(\bmod u_{r}^{\alpha_{r}}\right)$.

Применяем теперь Следствие 4 к системе $\mathrm{H}=\left(H_{1}, \ldots, H_{M} ; f^{(d)}, \ldots, f^{(d)}\right)$ и получаем, что множество всех длин циклов в $\operatorname{Str}\left(f^{(d)}, \mathbb{Z}_{N}^{*}\right)=\operatorname{Str}(\pi, H)$ - это совокупность всех различных чисел вида (50), где каждое $l_{r}$ пробегает все $t_{r}$ значений, указанных в (53).

б) Пусть $n-$ длина цикла в $\operatorname{Str}\left(f^{(d)}, \mathbb{Z}_{N}^{*}\right)$. Как показано в п. а), это означает, что существует вектор $\bar{j}=\left(j_{1}, \ldots, j_{M}\right)$, где $j_{r} \in I_{r}$ или $I_{r}^{(0)}$, для которого справедливо (53):

$$
n=\operatorname{HOK}\left(l_{1}, \ldots, l_{M}\right),
$$


и $\left(l_{r}\right)^{k_{r}}, r=1, \ldots, M,-$ фрагмент цикловой структуры $\operatorname{Str}\left(\pi_{r}, H_{r}\right)$. Следовательно, согласно Следствию 4,

$$
\star_{r=1}^{M}\left(l_{r}\right)^{k_{r}}=(n)^{K},
$$

где

$$
K=K(\bar{j})=\frac{k_{1} \ldots k_{M} l_{1} \ldots l_{M}}{\operatorname{HOK}\left(l_{1}, \ldots, l_{M}\right)}=\frac{k_{1} l_{1} \ldots k_{M} l_{M}}{n} .
$$

Найдём выражение для числителя этой дроби.

Предположим сначала, что $d \not \equiv 1\left(\bmod u_{r}^{\alpha_{r}}\right)$. Тогда, если $u_{r}$ нечётно, то $k_{r} l_{r}=1$ при $l_{r}=1$, а при $l_{r} \neq 1$

$$
k_{r} l_{r}= \begin{cases}a_{r} u_{r}^{j_{r}} \cdot \frac{\left(u_{r}-1\right) u_{r}^{\rho_{r}-1}}{a_{r}}=\left(u_{r}-1\right) u_{r}^{j_{r}+\rho_{r}-1}, & \text { когда } 1 \leqslant j_{r} \leqslant \alpha_{r}-\rho_{r}, \\ a_{r} \cdot \frac{u_{r}^{\rho_{r}}-1}{a_{r}}=u_{r}^{\rho_{r}}-1, & \text { когда } j_{r}=0 .\end{cases}
$$

Мы видим, что $k_{r} l_{r}=b_{r}$. Легко проверяется справедливость этого равенства также во всех случаях, когда $u_{r}=2$, и при $d \equiv 1\left(\bmod u_{r}^{\alpha_{r}}\right)$. Итак, $\prod_{r=1}^{M} k_{r} l_{r}=\prod_{r=1}^{M} b_{r}$ и, ввиду $(54)$,

$$
K=K(\bar{j})=\frac{1}{n} \prod_{r=1}^{M} b_{r}
$$

Пусть $\bar{j}^{(1)}, \ldots, \bar{j}^{(t)}$ - те и только те $M$-мерные векторы, которые приводят к представлению $n$ в виде (53). Как мы уже убедились, каждое такое представление даёт $K_{i}$ циклов длины $n$, где $K_{i}$ - число в $(55)$ - при значениях $b_{r}$, вычисляемых по координатам данного вектора $\bar{j}^{(i)}, i=1, \ldots, t$. Поэтому общее число циклов длины $n$ равно

$$
\sum_{i=1}^{t} K_{i}=\frac{1}{n} \sum \prod_{i=1}^{M} b_{r}
$$

где суммирование производится по всем возможным представлениям $n$ в виде (53).

Предложение доказано.

Полученные результаты дают конкретный способ нахождения $\operatorname{Str}\left(f^{(d)}, \mathbb{Z}_{N}^{*}\right)$ при любых $d$ и $N$.

1) Для $N$ в форме (40) находим набор простых чисел $U(d, N)$ из (42) и представление $(43)$ для $\operatorname{Ker}\left(f^{(d)}, \mathbb{Z}_{N}^{*}\right)$ в виде прямого произведения примарных циклических групп.

2) Для каждого $u_{r} \in U(d, N)$ находим числа $a_{r}=\operatorname{ord}\left(d, u_{r}\right)$ и $\rho\left(d, u_{r}\right)$ из $(17)$, если $u_{r}$ нечётно, и $v_{r}\left(\bar{d}, \alpha_{r}\right)$ из $(25)$ при $u_{r}=2$, и получаем структуpy $\operatorname{Str}\left(f^{(d)}, C_{u_{r}^{\alpha_{r}}}\right)$, используя формулу (18) или Предложения 8-10.

3) Берём по порядку все $M$-выборки фрагментов из $\operatorname{Str}\left(f^{(d)}, C_{u_{r} \alpha_{r}}\right), r=1, \ldots, M$, по одному из каждой структуры, и находим по формуле (50) для всех циклов в $\operatorname{Str}\left(f^{(d)}, \mathbb{Z}_{N}^{*}\right)$ их дли́ны, а по формуле (55) - общее число циклов каждой длины $n$.

Отметим некоторые следствия полученных результатов. Укажем формулу для максимальной длины цикла степенного отображения. 
Следствие 6. Пусть $d \geqslant 2, N \geqslant 2$ - произвольное натуральное число в форме $(40), U(d, N)$ - набор в (42), для которого построено прямое произведение (43), $U^{\prime}(d, N)$ - набор из $M^{\prime}$ чисел, $0 \leqslant M^{\prime} \leqslant M$, получающийся из $U(d, N)$ удалением всех $u_{r}$, для которых $d \equiv 1\left(\bmod u_{r}^{\alpha_{r}}\right)$. Тогда, если $M^{\prime} \neq 0$, то максимальная длина иикла в $\operatorname{Str}\left(f^{(d)}, \mathbb{Z}_{N}^{*}\right)$ есть

$$
n_{\max }=H O K\left(\Lambda_{1}, \Lambda_{2}, \ldots, \Lambda_{M^{\prime}}\right),
$$

где, для $u_{r} \in U^{\prime}(d, N)$,

$$
\Lambda_{r}= \begin{cases}a_{r} u_{r}^{\alpha_{r}-\rho_{r}}, & \text { если } u_{r} \text { нечётно, } \\ 2^{\alpha_{r}-v_{r}}, & \text { если } u_{r}=2, \alpha_{r} \geqslant 3, \\ 2, & \text { если } u_{r}=2, \alpha_{r}=2, d \equiv-1(\bmod 4),\end{cases}
$$

и $a_{r}=\operatorname{ord}\left(d, u_{r}\right), v_{r}=v\left(\bar{d}, \alpha_{r}\right)-$ число из $(25), \rho_{r}=\rho\left(d, u_{r}\right)-$ число из (17). Если $M^{\prime}=0$, mo $n_{\max }=1$.

Справедливость утверждения следует из Предложения 11, а также того факта (см. Предложения 7 и 10), что длина максимального цикла в $\operatorname{Str}\left(f^{(d)}, C_{u_{r}^{\alpha} r}\right)$ делится на длины всех остальных циклов.

Приведем формулы для цикловых структур в нескольких частных случаях, представляющих интерес для приложений.

Следствие 7. Пусть $d$ чётно, $N=p_{1} \ldots p_{m}-$ произведение $m$ различных простых чисел, $U(d, N)$ - набор (42) из $M$ простых чисел, по которому строится разложение (43). Предположим также, что

$$
d \not \equiv 1\left(\bmod u_{r}^{\alpha_{r}}\right) \quad \text { для любого } u_{r} \in U(d, N) .
$$

В этих условиях:

а) число $п$ является длиной цикла в $\operatorname{Str}\left(f^{(d)}, \mathbb{Z}_{N}^{*}\right)$ тогда и толъко тогда, когда

$$
n=\operatorname{HOK}\left(\left(a_{1} u_{1}^{j_{1}}\right)^{\delta_{1}},\left(a_{2} u_{2}^{j_{2}}\right)^{\delta_{2}}, \ldots,\left(a_{M} u_{M}^{j_{M}}\right)^{\delta_{M}}\right)
$$

где $a_{r}=\operatorname{ord}\left(d, u_{r}\right), r=1, \ldots, M$, для некоторьх $M$-мерных векторов $\bar{\delta}=$ $\left(\delta_{1}, \ldots, \delta_{M}\right) \in\{0,1\} u \bar{j}=\left(j_{1}, \ldots, j_{M}\right)$, где $0 \leqslant j_{r} \leqslant \alpha_{r}-\rho_{r} ;$

б) общее число ицклов длинь п равно

$$
\frac{1}{n} \sum \prod_{r=1}^{M} b_{r}, \quad b_{r}= \begin{cases}1, & \text { eсли } \delta_{r}=0 \\ u_{r}^{\rho_{r}}-1, & \text { если } j_{r}=0, \delta_{r}=1 \\ \left(u_{r}-1\right) u_{r}^{j_{r}+\rho_{r}-1}, & \text { если } j_{r}>0, \delta_{r}=1\end{cases}
$$

суммирование производится по всем возможным представлениям $n$ в виде (59). 
Действительно, ввиду чётности $d$, все числа $u_{r}$ в (42) нечётные. Поэтому, согласно Предложению 7 (формула (18)), числа $l_{r}$ в (50) принимают значения 1 или $a_{r}$, если $j_{r}=0$, и значения $a_{r} u_{r}^{j_{r}}$, если $\left.j_{r}>0\right)$; соответствующие фрагменты в $\operatorname{Str}\left(\pi_{r}, H_{r}\right)$, $H_{r}=C_{u_{r}^{\alpha_{r}}}$ принимают значения $(1),\left(a_{r}\right)^{\left(u_{r}^{\rho_{r}}-1\right) / a_{r}}$ или $\left(a_{r} u_{r}^{j_{r}}\right)^{\left(u_{r}-1\right) u_{r}^{\rho_{r}-1} / a_{r}}$.

Ясно тогда, что равенство (50) равносильно (59), если $\delta_{r}=0$ при тех и только тех $r$, при которых $l_{r}=1$. Далее, ввиду условия (58) и чётности $d$, число $b_{r}$ может принимать только значения $1, u_{r}^{\rho_{r}}-1$ или $\left(u_{r}-1\right) u_{r}^{j_{r}+\rho_{r}-1}, j_{r}=1, \ldots, \alpha_{r}-\rho_{r}$. Следовательно, справедливо также утверждение об общем числе циклов длины $n$.

Дваждъ простым называется такое простое число $p$, что $q=\frac{1}{2}(p-1)$ - также простое.

Следствие 8. Пусть выполнены условия Следствия 7, все числа $p_{1}, \ldots, p_{m}-\partial в а-$ жды простые и $U(d, N)=\left(q_{1}, q_{2}, \ldots, q_{M}\right)$, где $q_{r}=\frac{1}{2}\left(p_{r}-1\right), q_{r} \nmid d, r=1, \ldots, M$. Тогда иикловая структура $\operatorname{Str}\left(d, \mathbb{Z}_{N}^{*}\right)$ описывается следующим образом.

а) Число п является длиной цикла в том и только том случае, когда

$$
n=H O K\left(a_{1}^{\delta_{1}}, a_{2}^{\delta_{2}}, \ldots, a_{M}^{\delta_{M}}\right),
$$

где $a_{r}=\operatorname{ord}\left(d, q_{r}\right)$ для $q_{r} \in U(d, N), \bar{\delta}=\left(\delta_{1}, \ldots, \delta_{M}\right) \in\{0,1\}^{M}$.

б) Если п можно представить в виде (61) в точности при $t$ различных векто$\operatorname{pax} \bar{\delta}^{(1)}, \ldots, \bar{\delta}^{(t)}$, а $R_{1}^{(i)}-$ совокупность всех тех $r \in\{1, \ldots, M\}$, для которых $\delta_{r}^{(i)}=1$ в $\bar{\delta}^{(i)}=\left(\delta_{1}^{(i)}, \ldots, \delta_{M}^{(i)}\right)$. Тогда общее число циклов длинъь $n$ равно

$$
\frac{1}{n} \sum_{i=1}^{t} \prod_{r \in R_{1}^{(i)}}\left(q_{r}-1\right) .
$$

в) Максимальная длина иикла равна $n_{\max }=\operatorname{HOK}\left(a_{1}, a_{2}, \ldots, a_{M}\right)$.

Справедливость утверждения устанавливаем, учитывая соотношения (56)-(60).

\section{Список литературы}

1. Боревич З. И., Шафаревич И. Р., Теория чисел. Наука, Москва, 1964.

2. Brands S., Gill R., Cryptography, statistics and pseudorandomness. I. Probab. Math. Statist. (1995) 15, 101-114.

3. Brands S., Gill R., Cryptography, statistics and pseudorandomness. II. Probab. Math. Statist. (1996) 16, №1, 1-17.

4. Brennan J. J., Geist B., Analysis of iterated modular exponentiation: the orbits of $x^{\alpha} \bmod N$. Des., Codes and Cryptography (1998) 13, 229--245.

5. Chou W.-S., Shparlinski I. E., On the cycle structure of repeated exponentiation modulo a prime. J. Number Theory (2004) 107, 345-356.

6. Виноградов И. М., Основы теории чисел. Наука, Москва, 1981.

7. Ленг С., Алгебра. Мир, Москва, 1968. 
8. El-Mahassni E. D., On the distribution of the power generator over a residue ring for parts of the period. Rev. Mat. Comput. (2008) 21, 319-325.

9. Холл М., Теория групп. ИЛ, Москва, 1962.

10. Sha Min, On the cycle structure of repeated exponentiation modulo a prime power. arXiv:1101.3482v1[math.NT] 8 Jan 2011.

11. Vasiga T., Shallit J., On the iteration of certain quadratic maps over GF $(p)$. Discrete Math. (2004) 277, 219-240.

12. Wilson B., Power digraphs modulo n. Fibonacci Quart. (1998) 36, 229-239.

Статья поступила 01.08.2012. 